\title{
Herschel/HIFI observations of O-rich AGB stars: molecular inventory $\star, \star \star$
}

K. Justtanont ${ }^{1}$, T. Khouri ${ }^{2}$, M. Maercker ${ }^{3,16}$, J. Alcolea ${ }^{4}$, L. Decin ${ }^{5,2}$, H. Olofsson ${ }^{1}$, F. L. Schöier ${ }^{1, \star \star \star}$, V. Bujarrabal ${ }^{6}$, A. P. Marston ${ }^{7}$, D. Teyssier ${ }^{7}$, J. Cernicharo ${ }^{8}$, C. Dominik ${ }^{2,9}$, A. de Koter $^{2,10}$, G. Melnick ${ }^{11}$, K. M. Menten ${ }^{12}$, D. Neufeld ${ }^{13}$, P. Planesas ${ }^{4,14}$, M. Schmidt ${ }^{15}$, R. Szczerba ${ }^{15}$, and R. Waters ${ }^{2,5}$

${ }^{1}$ Onsala Space Observatory, Dept. Earth and Space Science, Chalmers University of Technology, 43992 Onsala, Sweden e-mail: kay. justtanont@chalmers.se

2 Sterrenkundig Instituut Anton Pannekoek, University of Amsterdam, Science Park 904, 1098 Amsterdam, The Netherlands

${ }^{3}$ University of Bonn, Argelander-Institut für Astronomie, Auf dem Hügel 71, 53121 Bonn, Germany

${ }^{4}$ Observatorio Astronómico Nacional (IGN), Alfonso XII N³, 28014 Madrid, Spain

5 Instituut voor Sterrenkunde, Katholieke Universiteit Leuven, Celestijnenlaan 200D, 3001 Leuven, Belgium

6 Observatorio Astronómico Nacional. Ap 112, 28803 Alcalá de Henares, Spain

7 European Space Astronomy Centre, ESA, PO Box 78, 28691 Villanueva de la Cañada, Madrid, Spain

${ }^{8}$ CAB, INTA-CSIC, Ctra de Torrejón a Ajalvir, km 4, 28850 Torrejón de Ardoz, Madrid, Spain

9 Department of Astrophysics/IMAPP, Radboud University Nijmegen, Nijmegen, The Netherlands

10 Astronomical Institute, Utrecht University, Princetonplein 5, 3584 CC Utrecht, The Netherlands

11 Harvard-Smithsonian Center for Astrophysics, Cambridge, MA 02138, USA

12 Max-Planck-Institut für Radioastronomie, Auf dem Hügel 69, 53121 Bonn, Germany

13 Johns Hopkins University, Baltimore, MD 21218, USA

14 Joint ALMA Observatory, Alonso de Córdova 3107, Vitacura, Santiago, Chile

15 N. Copernicus Astronomical Center, Rabiańska 8, 87-100 Toruń, Poland

16 European Southern Observatory, Karl Schwarzschild Str. 2, Garching bei München, Germany

Received 20 June 2011 / Accepted 14 November 2011

\section{ABSTRACT}

\begin{abstract}
Aims. Spectra, taken with the heterodyne instrument, HIFI, aboard the Herschel Space Observatory, of O-rich asymptotic giant branch (AGB) stars which form part of the guaranteed time key program HIFISTARS are presented. The aim of this program is to study the dynamical structure, mass-loss driving mechanism, and chemistry of the outflows from AGB stars as a function of chemical composition and initial mass.

Methods. We used the HIFI instrument to observe nine AGB stars, mainly in the $\mathrm{H}_{2} \mathrm{O}$ and high rotational CO lines. We investigate the correlation between line luminosity, line ratio and mass-loss rate, line width and excitation energy.

Results. A total of nine different molecules, along with some of their isotopologues have been identified, covering a wide range of excitation temperature. Maser emission is detected in both the ortho- and para- $\mathrm{H}_{2} \mathrm{O}$ molecules. The line luminosities of ground state lines of ortho- and para- $\mathrm{H}_{2} \mathrm{O}$, the high- $J \mathrm{CO}$ and $\mathrm{NH}_{3}$ lines show a clear correlation with mass-loss rate. The line ratios of $\mathrm{H}_{2} \mathrm{O}$ and $\mathrm{NH}_{3}$ relative to $\mathrm{CO} J=6-5$ correlate with the mass-loss rate while ratios of higher CO lines to the $6-5$ is independent of it. In most cases, the expansion velocity derived from the observed line width of highly excited transitions formed relatively close to the stellar photosphere is lower than that of lower excitation transitions, formed farther out, pointing to an accelerated outflow. In some objects, the vibrationally excited $\mathrm{H}_{2} \mathrm{O}$ and $\mathrm{SiO}$ which probe the acceleration zone suggests the wind reaches its terminal velocity already in the innermost part of the envelope, i.e., the acceleration is rapid. Interestingly, for R Dor we find indications of a deceleration of the outflow in the region where the material has already escaped from the star.
\end{abstract}

Key words. stars: AGB and post-AGB - circumstellar matter - stars: late-type - infrared: stars - line: identification

\section{Introduction}

Asymptotic giant branch (AGB) stars represent a late stage of stellar evolution when the nuclear burning in the core has ceased. The dominant factor governing the rest of their evolution is the

\footnotetext{
* Herschel is an ESA space observatory with science instruments provided by European-led Principal Investigator consortia and with important participation from NASA.

$\star \star$ Appendix A is available in electronic form at

http://www . aanda.org

$\star \star \star$ Deceased.
}

mass loss from the surface (Habing 1996). Presently, the initial mechanism(s) driving the wind which leads to dust formation in O-rich stars is not fully understood. Model calculations of dynamics of the photosphere of AGB stars show that shockwaves arising from pulsation can levitate the material and produce an extended atmosphere (e.g., Bowen 1988; Hoefner \& Dorfi 1992, 1997). The latter authors showed that the models lead to dust formation in the atmosphere. Although the efficiency of dust formation in the outflow of AGB stars has been questioned (see e.g., Woitke 2006), Höfner (2008) proposed the wind is driven by micron-size grains which are Fe-poor. Mattsson et al. (2008) 
also show that for AGB stars with a low metallicity, pulsation can lead to an intense mass-loss rate. Due to the larger cross sections of dust grains compared to molecules, dust efficiently absorbs stellar radiation and is accelerated outwards, dragging the gas along with it (Goldreich \& Scoville 1976; Justtanont et al. 1994; Decin et al. 2006; Ramstedt et al. 2008). This effectively establishes a circumstellar envelope around the star.

The circumstellar envelope of an AGB star is an active site for chemistry. The slowly expanding wind $\left(v_{\mathrm{e}} \sim 10-15 \mathrm{~km} \mathrm{~s}^{-1}\right)$ produced by a constant, isotropic mass-loss rate, is an ideal laboratory to study physical and chemical processes during stellar evolution. In thermal equilibrium, the relative abundance of carbon and oxygen determines the composition of the molecules and dust species formed. For a carbon-rich star with $\mathrm{C} / \mathrm{O}>1$, carbonaceous molecules form as well as amorphous carbon and $\mathrm{SiC}$ dust after all the oxygen is locked up in the most abundant trace molecule $\mathrm{CO}$. In contrast, for a star with $\mathrm{C} / \mathrm{O}<1$, i.e., O-rich (M-type) AGB stars, $\mathrm{H}_{2} \mathrm{O}$ and $\mathrm{CO}$ are the main gas components, along with silicate dust. However, close to the photosphere, shock waves due to stellar pulsations and/or the stellar radiation field can induce non-thermal equilibrium, rendering this simple picture more complicated (Cherchneff 2006). As an example, the canonical C-rich AGB star, IRC+10216 has been found to exhibit $\mathrm{H}_{2} \mathrm{O}$ emission (Melnick et al. 2001; Hasegawa et al. 2006; Decin et al. 2010a). Data obtained with HerschelHIFI have recently shown that $\mathrm{H}_{2} \mathrm{O}$ is quite prevalent in C-stars (Neufeld et al. 2011).

HIFISTARS is the guaranteed time key program aimed at studying circumstellar envelopes around evolved stars using the heterodyne instrument (HIFI, de Graauw et al. 2010) aboard the Herschel Space Observatory (Pilbratt et al. 2010). The program aims to study stars with a large range of mass-loss rates $\left(10^{-7}-10^{-4} M_{\odot} \mathrm{yr}^{-1}\right)$, differing chemistry $(\mathrm{C} / \mathrm{O}<1, \mathrm{C} / \mathrm{O} \sim 1$, $\mathrm{C} / \mathrm{O}>1$ ) and initial mass (low- and intermediate mass stars and supergiants), as well as different evolutionary (AGB to planetary nebula) phases. In this paper, we report all the observations done on O-rich AGB stars in our program. Observations and data reduction are presented in Sect. 2 and the lines detected and the first-cut interpretation are discussed in Sect. 3. The results are summarised in Sect. 4.

\section{Observations}

Our HIFI observations were carried out using dual-beam switch mode with a throw of $3^{\prime}$ and a slow chopping. The full bandwidth of HIFI of $4 \mathrm{GHz}$ was utilized using the wide-beamspectrometer backend. A total of 16 different frequency settings have been chosen to cover a number of expected strong lines of $\mathrm{H}_{2} \mathrm{O}$ and $\mathrm{CO}$ for the purpose of sampling different regions of the warm circumstellar envelope in these objects. The data were calibrated using the standard Herschel pipeline, HIPE, and reprocessed for those which had been processed with the pipeline version earlier than 4.0. Data were taken using two orthogonal polarizations: horizontal and vertical. A resulting spectrum is an average of these two polarizations which is then rebinned to a $1 \mathrm{~km} \mathrm{~s}^{-1}$ resolution (Figs. A.1-A.12). However, a number of spectra in the THz-band (HIFI bands 6 and 7) were affected by the ripples, especially in the $v$-polarization. These are thought to be due to standing waves in the hot electron bolometer (HEB) mixers. In these cases, we rebinned only the $h$-polarization spectrum. The spectra have been corrected for the beam efficiency, $\eta_{\mathrm{mb}}$

$\eta_{\mathrm{mb}}=\eta_{\mathrm{mb}, 0} \exp \left(-(4 \pi \sigma / \lambda)^{2}\right)$
Table 1. O-rich AGB stars in the sample.

\begin{tabular}{lccccc}
\hline \hline Name & RA & Dec & obs & $\begin{array}{c}D \\
(\mathrm{pc})\end{array}$ & $\begin{array}{c}\dot{M} \\
\left(M_{\odot} \mathrm{yr}^{-1}\right)\end{array}$ \\
\hline IRC+10011 & 010626.0 & +123553.0 & 15 & 740 & $1.9 \mathrm{E}-5^{(1)}$ \\
$o$ Cet & 021920.8 & -025839.5 & 9 & 107 & $2.5 \mathrm{E}-7^{(1)}$ \\
IK Tau & 035328.9 & +112421.7 & 15 & 260 & $4.5 \mathrm{E}-6^{(1)}$ \\
R Dor & 043645.6 & -620437.8 & 9 & 59 & $2.0 \mathrm{E}-7^{(2)}$ \\
TX Cam & 050050.4 & +561052.6 & 9 & 380 & $6.5 \mathrm{E}-6^{(1)}$ \\
W Hya & 134902.0 & -282203.5 & 16 & 77 & $7.8 \mathrm{E}-8^{(1)}$ \\
AFGL 5379 & 174424.0 & -315535.5 & 8 & 580 & $2.0 \mathrm{E}-4^{(3)}$ \\
OH 26.5+0.6 & 183732.5 & -052359.2 & 8 & 1370 & $2.6 \mathrm{E}-4^{(3)}$ \\
R Cas & 235824.9 & +512319.7 & 9 & 106 & $4.0 \mathrm{E}-7^{(1)}$ \\
\hline
\end{tabular}

Notes. Indicated is the number of frequency settings observed for each object.

References. The quoted distance and mass-loss rates are taken from (1) de Beck et al. (2010); (2) Maercker et al. (2009); and (3) Justtanont et al. (2006).

where $\sigma$ is the surface accuracy $(=3.8 \mu \mathrm{m}), \lambda$ is the wavelength of the transition and $\eta_{\mathrm{mb}, 0}$ is a correction factor of 0.76 , except for the frequency range of 1120-1280 GHz (HIFI band 5) where this value is 0.66 (Olberg 2010, http://herschel.esac.esa.int/Docs/ TechnicalNotes/HIFI-Beam-Efficiencies-17Nov2010. pdf).

In three objects, IRC+10011, IK Tau, and W Hya, we observe a large number of frequency settings $(15,15$ and 16 , respectively), with the intention of using them as templates for other objects with high, intermediate and low mass-loss rates, respectively. In order to study a larger sample of stars, we also observed 6 further objects with fewer frequency settings selected from the original settings (Table 1) which include many diagnostic lines (see Table A.1).

In order to estimate the integrated line intensity, a Gaussian fit was performed in IDL. Some lines are flat-topped and hence the line intensity was estimated using a rectangular (rhombic) profile. Due to the observing mode used, it is not possible to recover the true continuum level of the spectra. However, the estimated line intensities are not affected by this since we subtract the continuum prior to calculating them. Our error estimates reflect only the noise in the baseline. Other uncertainties such as the assumption that a line is Gaussian are not included. However, independent measurements of the line intensity by summing the area under the line agree within twice the estimated uncertainties listed in Table A.1. The absolute flux calibration error is expected to be $\sim 30 \%$ in the HIFI bands 6 and 7 and less $(\sim 15 \%)$ at lower frequencies.

\section{Molecular lines}

We identify a total of 9 different molecular species as well as associated isotopologues (Table A.1). Most of the lines are in the ground vibrational state. However, for $\mathrm{H}_{2} \mathrm{O}$ and $\mathrm{SiO}$, vibrationally excited lines are also seen indicating that the lines originate from the hotter part of the envelope (Sect. 3.3). Many of the settings observed in the star IK Tau have been presented by Decin et al. (2010b) and are included here for completeness of our sample, along with new observations.

It has been postulated that $\mathrm{H}_{2} \mathrm{O}$ is one of the main molecular coolants in the circumstellar envelopes of O-rich AGB stars, along with CO (Goldreich \& Scoville 1976). This was confirmed 
by observations by the Infrared Space Observatory (ISO) of these stars which show numerous strong lines of both these molecules (e.g. Barlow et al. 1996; Neufeld et al. 1996). We detected a total of $23 \mathrm{H}_{2} \mathrm{O}$ lines in all the three main isotopologues covering the excitation temperature from 30 to $2350 \mathrm{~K}$. These lines probe the full extent of the circumstellar envelope and due to HIFI resolution, the lines are well resolved, enabling us to study the dynamics of the dust-driven wind. It should be noted that the $\mathrm{H}_{2} \mathrm{O} 1_{1,0}-1_{0,1}$ vibrationally excited line at $658.0 \mathrm{GHz}$ is likely to be a maser, as well as the $620.7 \mathrm{GHz}\left(5_{3,2}-4_{4,1}\right)$ line (see Sect. 3.2). This latter line has previously been reported in the supergiant VY CMa (Harwit et al. 2010). As for CO, we detected a total of 8 transitions in three isotopologues.

Other molecules are also identified, such as $\mathrm{NH}_{3}, \mathrm{SiO}, \mathrm{HCN}$, $\mathrm{SO}, \mathrm{SO}_{2}$ and $\mathrm{OH}$. These molecules have already been detected in HIFI spectra of AGB and post-AGB stars (Bujarrabal et al. 2010; Decin et al. 2010b; Menten et al. 2010; Justtanont et al. 2010; Schöier et al. 2011). In AFGL 5379, a line is detected at $1196.010 \mathrm{GHz}$ which can be attributed to the $\mathrm{H}_{2} \mathrm{~S}(3,1,2)-(2,2,1)$ transition. This line is not seen in the other stars in our sample, however it is present in the supergiant VY CMa (Alcolea 2011, in prep.). A line is also seen in the spectrum of $\mathrm{OH} 26.5+0.6$ at $1114.431 \mathrm{GHz}$ which corresponds to the vibrationally excited $\mathrm{SiS} J=62-61$ transition. Although, with the high upper energy level of $1918.3836 \mathrm{~cm}^{-1}$, we classify this line as unlikely.

Since HIFI employs a double-side band mode, there is a possibility of ambiguity in classifying a line. The line at $1112.833 \mathrm{GHz}$ in the lower side-band due to ${ }^{29} \mathrm{SiO} J=$ 26-25 coincides with the $\mathrm{H}_{2} \mathrm{O} \quad 11_{6,6}-12_{3,9}$ at $1101.130 \mathrm{GHz}$. Considering the upper energy levels and the expected line strengths of the two, we conclude that the line is likely due to ${ }^{29} \mathrm{SiO}$.

\subsection{Line intensities}

All objects show strong emission due to $\mathrm{H}_{2} \mathrm{O}$ and $\mathrm{CO}$ molecules (Fig. 1). The OH/IR stars, OH 26.5+0.6, AFGL 5379 and IRC +10011 , show very strong emission of both ground state ortho- and para- $\mathrm{H}_{2} \mathrm{O}$ lines compared to that of $\mathrm{CO} J=6-5$. Despite the high mass-loss rates in these OH/IR stars, the CO $J=16-15$ is very weak or not detected. This may be partly due to the attenuation of stellar light by dust, preventing the excitation of this line. In $o$ Cet, the CO lines are much stronger than other molecular species. This is likely due to the white-dwarf companion with its hard X-ray flux (O'Dwyer et al. 2003) photodissociating $\mathrm{H}_{2} \mathrm{O}$ molecules in the circumstellar envelope of the primary star. In all the stars, we detect both ${ }^{12} \mathrm{CO}$ and ${ }^{13} \mathrm{CO}$. The line ratios of the corresponding transitions vary from 1.5 in stars with high mass-loss rate to up to 10 in stars with lower mass-loss rates.

We detected both the ortho- and para- $\mathrm{H}_{2} \mathrm{O}$ as well as the isotopologues and vibrationally excited lines. A discussion on isotopic ratios of detected lines is presented by Silva et al. (2011, in prep.). A detailed modelling of radiative transfer of stars with low mass-loss rates is in progress (Maercker et al. 2011, in prep.). The highest excitation lines seen in our spectra are the vibrationally excited $2_{12}-1_{01}$ and the vibrationally excited ground state lines of both ortho- and para- $\mathrm{H}_{2} \mathrm{O}$ with excitation energies in excess of $2000 \mathrm{~K}$ (Table A.1). These lines are likely radiatively pumped.

In Fig. 2, we plot the line luminosity as a function of mass-loss rate for $\mathrm{CO} J=6-5,10-9$, and the ground-state lines of both ortho- and para- $\mathrm{H}_{2} \mathrm{O}$, as well as for $\mathrm{NH}_{3} 1_{0}-0_{0}$.

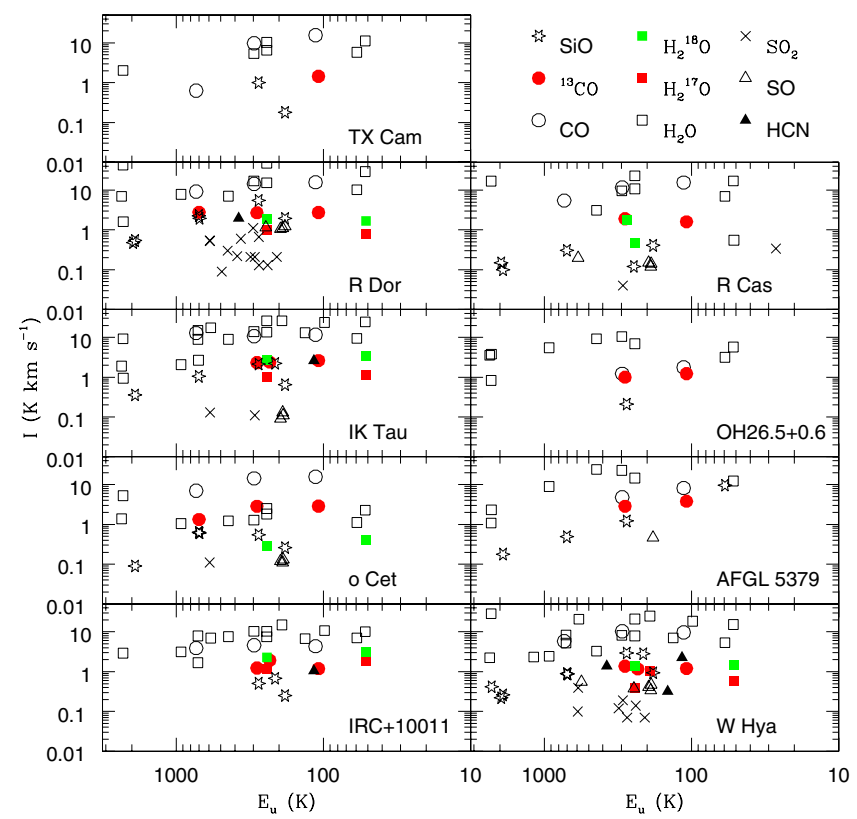

Fig. 1. Integrated line intensities for all stars in the sample. ${ }^{12} \mathrm{CO}$ : black open circles; ${ }^{13} \mathrm{CO}$ red filled circles; $\mathrm{H}_{2}^{16} \mathrm{O}$ : open squares; $\mathrm{H}_{2}^{17} \mathrm{O}$ : red filled squares; $\mathrm{H}_{2}^{18} \mathrm{O}$ : green filled squares; $\mathrm{SiO}$ : stars; $\mathrm{SO}$ : open triangles; $\mathrm{SO}_{2}: \times ; \mathrm{HCN}$ : filled triangles.

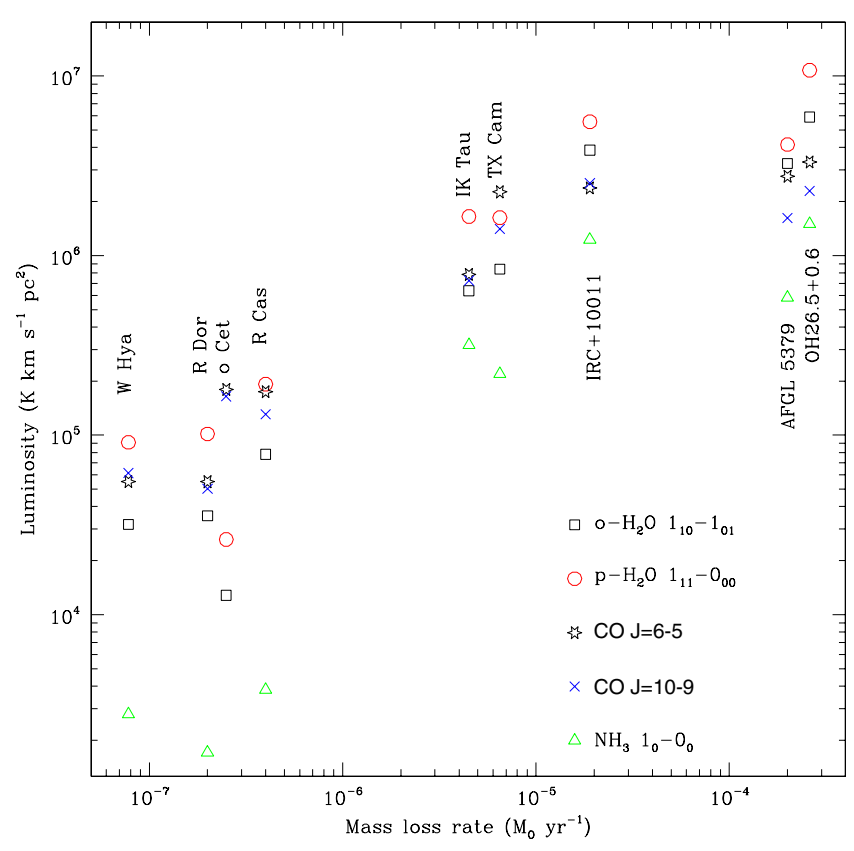

Fig. 2. A plot of the line luminosity (squares: ortho- $\mathrm{H}_{2} \mathrm{O} 1_{1,0}-1_{0,1}$, circles: para- $\mathrm{H}_{2} \mathrm{O} 1_{1,1}-0_{0,0}$, stars: $\mathrm{CO} J=6-5, \times$ : $\mathrm{CO} J=10-9$, triangles: $\mathrm{NH}_{3} 1_{0}-0_{0}$ ) as a function of the published mass-loss rate show a strong correlation between the two parameters.

The distances and mass-loss rates are taken from de Beck et al. (2010); Maercker et al. (2009); Justtanont et al. (2006) (see Table 1). There is a strong positive correlation between individual $\mathrm{H}_{2} \mathrm{O}$ and $\mathrm{CO}$ line luminosities, i.e., the slopes for the mass-loss rate up to $\sim 10^{-5} M_{\odot} \mathrm{yr}^{-1}$ are betwen $0.7-0.8$. There is one exception regarding the $\mathrm{H}_{2} \mathrm{O}$ luminosity in $o$ Cet which is much lower than for the CO. This is likely due to $\mathrm{H}_{2} \mathrm{O}$ being photodissociated by the binary companion. The flattening off of 
this relation at $\dot{M} \sim 3 \times 10^{-5} M_{\odot} \mathrm{yr}^{-1}$ is consistent with relations by de Beck et al. (2010).

Another molecule readily detected in circumstellar envelopes of AGB stars is $\mathrm{SiO}$ (e.g., Bujarrabal et al. 1994; Olofsson et al. 1998). Here, we identified three silicon isotopes. The vibrationally excited lines of high- $J$ transitions are also seen.

SO has been observed in a number of AGB stars covering a wide range in mass-loss rates. The estimated abundance is a few $10^{-7}$ up to $10^{-6} M_{\odot} \mathrm{yr}^{-1}$ (Omont et al. 1993; Bujarrabal et al. 1994). However, we only observed $\mathrm{SO}$ and $\mathrm{SO}_{2}$ lines in our HIFI frequency range in stars with low mass-loss rates. From ISO observations, $\mathrm{SO}_{2}$ absorption is detected towards stars with optically thin envelopes (Yamamura et al. 1999), while this is not seen in optically thick shells. SO is thought to be formed mainly via $\mathrm{S}+\mathrm{CO}$ and to a lesser extent via $\mathrm{S}+\mathrm{OH}$ (Cherchneff 2006) and is expected to be present throughout the envelope. It follows then that the formation of $\mathrm{SO}_{2}$ is via $\mathrm{SO}+\mathrm{OH}$. The best example for $\mathrm{SO}$ and $\mathrm{SO}_{2}$ can be seen in our spectrum of R Dor (Fig. A.6). In this object, the line fluxes of the SO lines are about an order of magnitude larger than that of $\mathrm{SO}_{2}$. In the other stars where both molecules are observed, the flux ratios are less than 5. The only other S-bearing molecule seen in our spectra is $\mathrm{H}_{2} \mathrm{~S}$ in AFGL 5379 at $1196.0 \mathrm{GHz}$. This line is absent in all the other stars in our sample, compare to a more ubiquitous presence of this molecule observed from the ground in OH/IR stars (Omont et al. 1993).

We detected the $\mathrm{OH}$ triplet line at $1834.7 \mathrm{GHz}$, which is very strong in the two extreme OH/IR stars, in agreement with the fact that these stars emit strongly in the $\mathrm{OH} 1612 \mathrm{MHz}$ maser (e.g., Sevenster et al. 2001). The spectrum of IRC+10011 is contaminated by ripples (standing waves in the HEB mixers) preventing us from confirming the presence of the $\mathrm{OH}$ line.

To study the effects of mass-loss rate on the excitation, we plot line intensity ratios of CO $J=16-15$ and $J=10-9$ relative to the $J=6-5$ (Fig. 3 middle panel). These ratios appear to be constant over four orders of magnitude of mass-loss rates, indicating that the density is higher than critical density of these transitions, i.e., the lines are thermalized in the emitting region. The line ratios of the ground state lines of both orthoand para- $\mathrm{H}_{2} \mathrm{O}$ with $\mathrm{CO}$, on the other hand, show an increasing trend as a function of mass-loss rate with the para- $\mathrm{H}_{2} \mathrm{O}$ having a consistently higher ratio than that of the ortho- $\mathrm{H}_{2} \mathrm{O}$. This same increasing trend can be seen between the $\mathrm{NH}_{3} 1_{0}-0_{0}$ and mass-loss rates, indicative of similar excitation conditions for both molecules. The top panel of Fig. 3 shows the $\mathrm{SO} / \mathrm{CO}$ and $\mathrm{SO}_{2} / \mathrm{CO}$ ratios. Here, a decreasing trend for the line ratio with the mass-loss rate can be seen. From the HIFI observations, both $\mathrm{SO}$ and $\mathrm{SO}_{2}$ are not detected in stars with a mass-loss rate higher than $10^{-5} M_{\odot} \mathrm{yr}^{-1}$. It is therefore not possible to confirm the tight correlation seen by Olofsson et al. (1998) between CO $(J=1-0)$ and SO $\left(J_{\mathrm{k}}=3_{2}-2_{1}\right)$ line fluxes. Omont et al. (1993), however, detected low excitation lines of $\mathrm{SO}$ and $\mathrm{SO}_{2}\left(T_{\mathrm{ex}} \leq 55 \mathrm{~K}\right)$ in $14 \mathrm{OH} / \mathrm{IR}$ stars, including three stars in our sample (IRC+10011, IK Tau and $\mathrm{OH} 26.5+0.6)$. In optically thick envelopes, the high excitation levels of the sulphur-bearing molecules in the HIFI range $\left(T_{\text {ex }} \geq 200 \mathrm{~K}\right)$ are simply not sufficiently excited.

\section{2. $\mathrm{H}_{2} \mathrm{O}$ masers}

Maser emission has been observed toward a large number of evolved stars. Its signature includes an anomalously strong line intensity and a narrow line width due to maser amplification. In

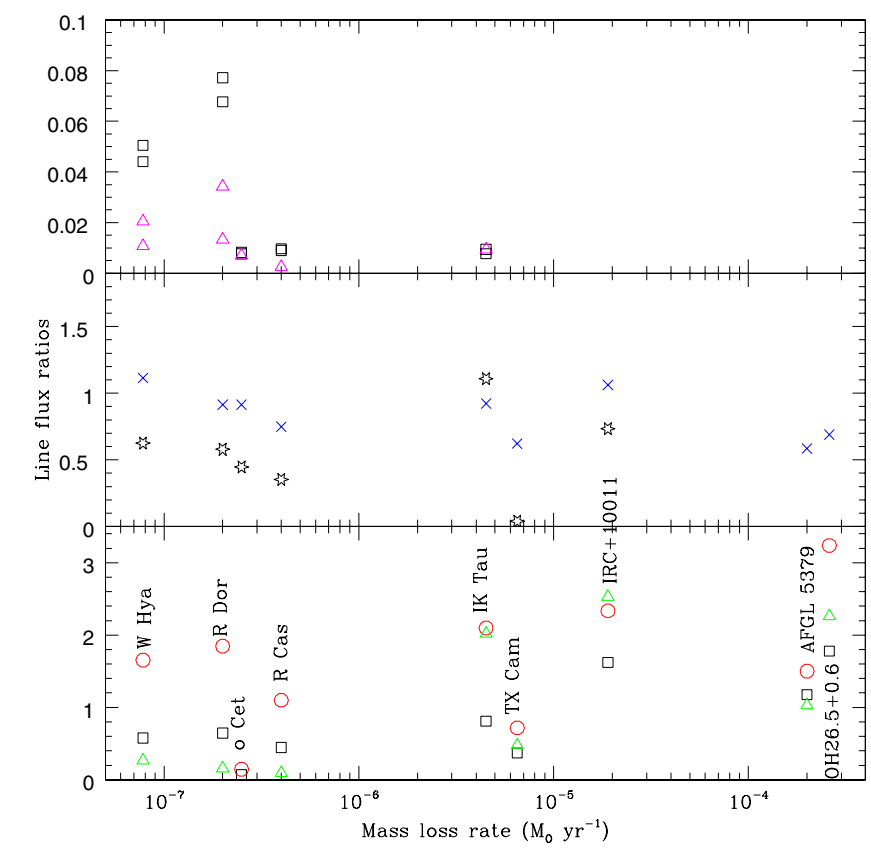

Fig. 3. A plot of the line ratios relative to the integrated intensity of the $\mathrm{CO} J=6-5$ as a function of mass-loss rates. In the lower panel: for ortho- $\mathrm{H}_{2} \mathrm{O} 1_{1,0}-1_{0,1}$ (squares), para- $\mathrm{H}_{2} \mathrm{O} 1_{1,1}-0_{0,0}$ (circles), $\mathrm{NH}_{3} 1_{0}-0_{0}$ (triangles) scaled up by a factor of 5. The middle panel shows CO $J=10-9(\times), \mathrm{CO} J=16-15$ (stars) and in the upper panel: SO $\left(13_{13}-12_{12}\right) 559.3$ and SO $\left(13_{14}-12_{13}\right) 560.2 \mathrm{GHz}$ (triangles) and $\mathrm{SO}_{2}\left(21_{6,16}-21_{5,17}\right) 558.4$ and $\mathrm{SO}_{2}\left(37_{1,37}-36_{0,36}\right) 659.4 \mathrm{GHz}$ (squares).

some instances, a maser can be seen in a narrow peak in the blue wing.

In three objects (W Hya, IK Tau, and IRC+10011), we observe the $620.7 \mathrm{GHz}$ transition of ortho- $\mathrm{H}_{2} \mathrm{O} 5_{3,2}-4_{4,1}$ which is predicted to be masing (Neufeld \& Melnick 1991). The maser line is brighter compared to the thermal excited lines and tends to have a narrower line profile as the region of coherent velocity required to produce maser emission is generally small. In W Hya, the maser line is narrow compared to the thermally excited lines of $\mathrm{H}_{2} \mathrm{O} 1_{1,0}-1_{0,1}$ and $\mathrm{CO} J=6-5$, indicating it comes from a region close to the star. In IK Tau, the line exhibits a double-peak profile which has a line width slightly narrower than that of the $556.9 \mathrm{GHz}$ ground-state ortho- $\mathrm{H}_{2} \mathrm{O}$ and the CO line. In IRC+10011, however, the line is of the same width as the ground state $\mathrm{H}_{2} \mathrm{O}$ and $\mathrm{CO} J=6-5$ lines, and is thought to arise from thermal emission, but it also has a strong blue component due to the masing action (see Fig. 4 lower panels). This is in line with the expectation that the $620.7 \mathrm{GHz}$ line is masing at high $\left(\sim 10^{3} \mathrm{~K}\right)$ temperature (Neufeld \& Melnick 1991). The maser emission appears to arise from the blue-shifted part of the spectrum, i.e., in the front side of the envelope. Neufeld \& Melnick (1991) also predicted a maser line at $970.3 \mathrm{GHz}$ from para- $\mathrm{H}_{2} \mathrm{O}\left(5_{2,4}-4_{3,1}\right)$ which we observed in W Hya and IK Tau with similar line widths and profiles as for the $620.7 \mathrm{GHz}$ maser. In IRC+10011, we did not detect a maser peak (Fig. 4 upper panels), and the line is about the same brightness as for the ground transition line at $556.9 \mathrm{GHz}$ (Table A.1), hence the maser action appears to be quenched in this object.

In one of the frequency settings, we detected the vibrationally excited $\mathrm{H}_{2} \mathrm{O} 1_{1,0}-1_{0,1}$ line at $658.0 \mathrm{GHz}$ which is likely masing (Fig. 5). In all the objects with low and intermediate mass-loss rates, the maser line is markedly narrow compared 


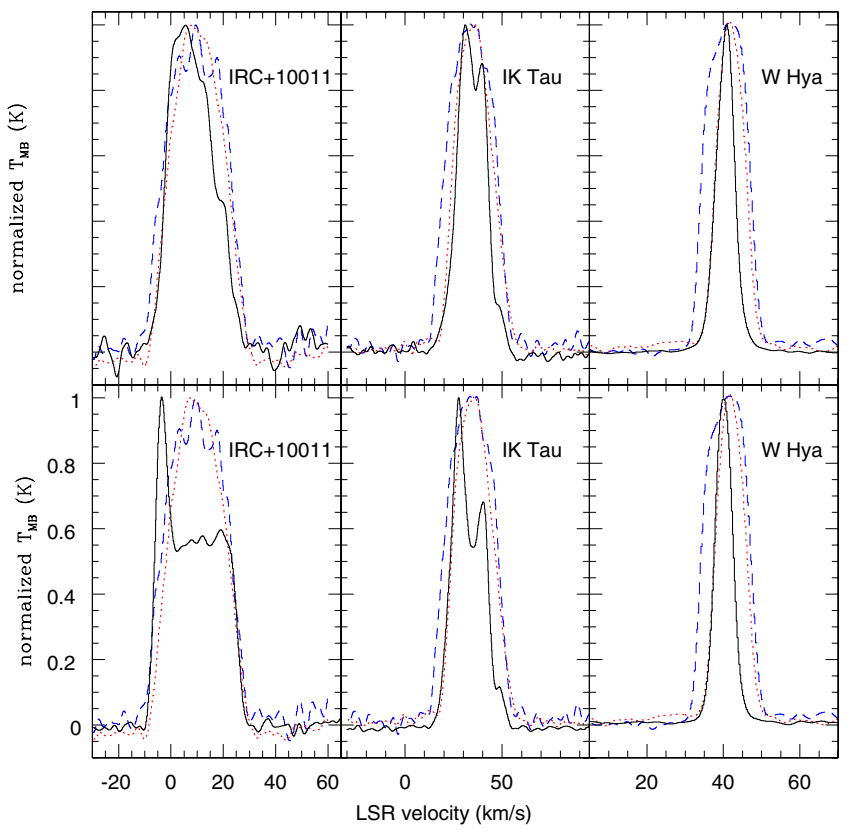

Fig. 4. The lower panels show a comparison of the normalized line profile of the $620.7 \mathrm{GHz} \mathrm{H}_{2} \mathrm{O} 5_{3,2}-4_{4,1}$ line (solid line) with the ground state ortho- $\mathrm{H}_{2} \mathrm{O} 1_{1,0}-1_{0,1}$ line at $556.9 \mathrm{GHz}$ (dotted red line) and the CO $J=6-5$ (dashed blue line). The upper panels show the $970.3 \mathrm{GHz}$ $\mathrm{H}_{2} \mathrm{O} 5_{2,4}-4_{3,1}$ (solid) with same the $\mathrm{H}_{2} \mathrm{O}$ ground state and $\mathrm{CO}$ lines.

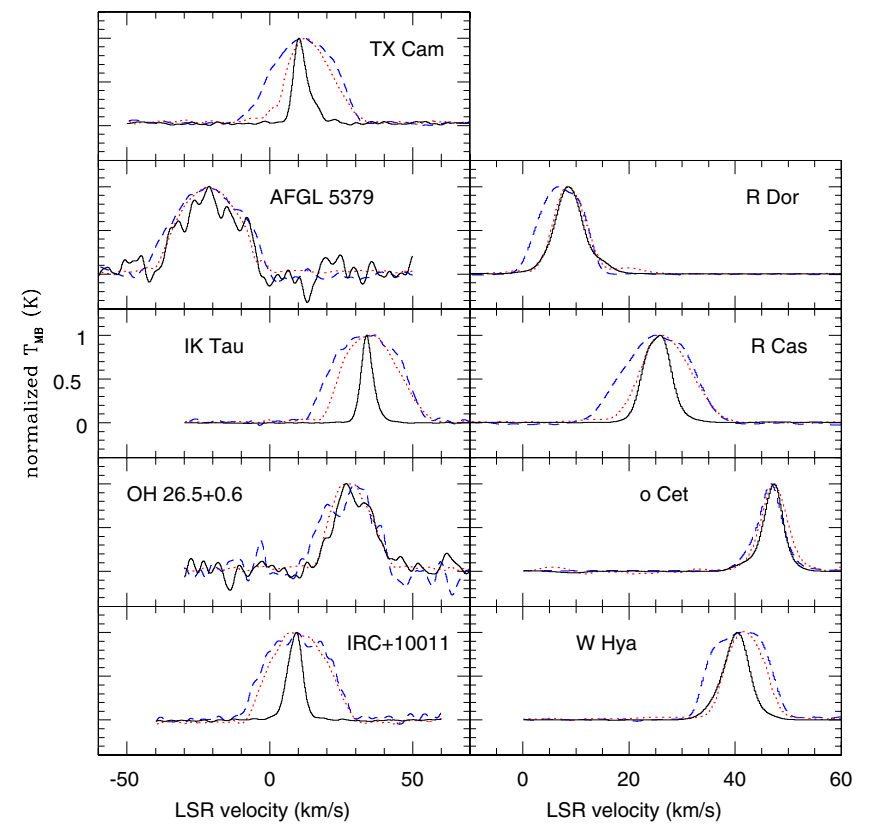

Fig. 5. A comparison of the normalized line profile of the $658.0 \mathrm{GHz}$ $\mathrm{H}_{2} \mathrm{O}$ line (solid line) with the ground state ortho- $\mathrm{H}_{2} \mathrm{O}$ line at $556.9 \mathrm{GHz}$ (dotted red line) and the $\mathrm{CO} J=6-5$ (dashed blue line).

to the $556.9 \mathrm{GHz}$ line except for $o$ Cet, where both lines have comparable widths. This indicates, again, that the maser line in the majority of the stars originates close to the star where the wind has not yet reached the terminal velocity. The intrinsic line intensity supports the fact that the line is masing, i.e., the line intensity of the vibrationally excited line is very bright, compared with the ground state line at $556.9 \mathrm{GHz}$. However, in extreme $\mathrm{OH} / \mathrm{IR}$ stars $(\mathrm{OH} 26.5+0.6$ and AFGL 5379), the widths of the ground and vibrationally excited lines are comparable and the flux of the vibrationally excited line is small relative to the ground state line. It is unlikely then in these two objects that the line is masing. This is probably due to the high density quenching maser action in the inner part of the envelope.

We also observe the vibrationally excited line of para$\mathrm{H}_{2} \mathrm{O} 1_{1,1}-0_{0,0}$ at $1205.8 \mathrm{GHz}$. Unlike the vibrationally excited $1_{1,0}-1_{0,1}$ ortho- $\mathrm{H}_{2} \mathrm{O}$, this line does not appear to be masing when comparing with the same transition in the ground vibrational state at $1113.3 \mathrm{GHz}$ as the vibrationally excited line is much weaker.

\subsection{Expansion velocity of circumstellar envelops}

Once dust grains condense in the outflow of AGB stars, the wind acceleration mechanism is thought to be due to radiation pressure on the dust grains, which drags the gas as they move away from the central star (e.g., Goldreich \& Scoville 1976; Habing et al. 1994). Lines with a high excitation temperature are expected to be emitted in regions close to the star, where the wind has not yet reached its terminal velocity. Consequently, these lines would be observed to be narrower than lines with lower excitation temperatures, emitted from farther out in the envelope. Justtanont et al. (1994) demonstrated that in a radiative transfer calculation for successively higher- $J$ CO lines, the warmer regions of the emitting zone are being probed which have smaller line widths than for the lowest CO transition. However, observations of maser lines which probe regions close to the central star revealed that the wind acceleration is not as fast as predicted by the dust-drag wind (Chapman et al. 1994). In Fig. 6, we plot the expansion velocities for the lines detected in our spectra, which probe the warm region as well as the region close to the central star. Here, the observed expansion velocity is a measure of half the width at the baseline level. The uncertainty of the expansion velocity is $\sim 15-30 \%$. In most cases, there is a trend that lower excitation lines have wider observed line profiles, indicating they come from regions where the wind is close to the previously determined expansion velocity from ground-based observations of low- $J$ CO lines. This indicates that there is a velocity gradient in the circumstellar outflow. One caveat is that for $\mathrm{H}_{2} \mathrm{O}$ lines, the profile can be heavily absorbed in the blue wing, leading to slightly lower measured expansion velocities. This can be seen in Fig. 6 which shows that the measured expansion velocity of $\mathrm{CO} J=6-5$ is larger than that for the ground state of both orthoand para- $\mathrm{H}_{2} \mathrm{O}$. We observe isotopic lines of $\mathrm{H}_{2} \mathrm{O}$, and a trend can be seen that $v\left(\mathrm{H}_{2}^{16} \mathrm{O}\right)>v\left(\mathrm{H}_{2}^{17} \mathrm{O}, \mathrm{H}_{2}^{18} \mathrm{O}\right)$. This may be due to the fact that the less abundant species are more prone to photodissociation from the interstellar radiation hence we see molecules close to the central star where the gas has not yet reached the terminal velocity.

For all stars, 3 CO lines have been targeted: $J=6-5,10-9$ and 16-15. The widths of these lines show the trend of increasing velocity for the lower excitation line, in agreement with the acceleration. However, the $J=16-15$ line is not seen towards the two extreme OH/IR stars, OH 26.5+0.6 and AFGL 5379, possibly due to the dust attenuation of stellar radiation, preventing the excitation of this line. We readily detected the ${ }^{13} \mathrm{CO}$ lines in our frequency settings as well and in most cases, the line widths of these two isotopes are comparable.

The line widths of $\mathrm{SO}$ and $\mathrm{SO}_{2}$ are narrower than those from $\mathrm{CO}$ and $\mathrm{H}_{2} \mathrm{O}$. Due to the low abundance, the lines are not thought to be optically thick, hence the reason why the lines are narrower is likely because they originate from the inner region of the envelope. It can be that these molecules are also destroyed closer in the central star by interstellar UV radiation, compared to the 


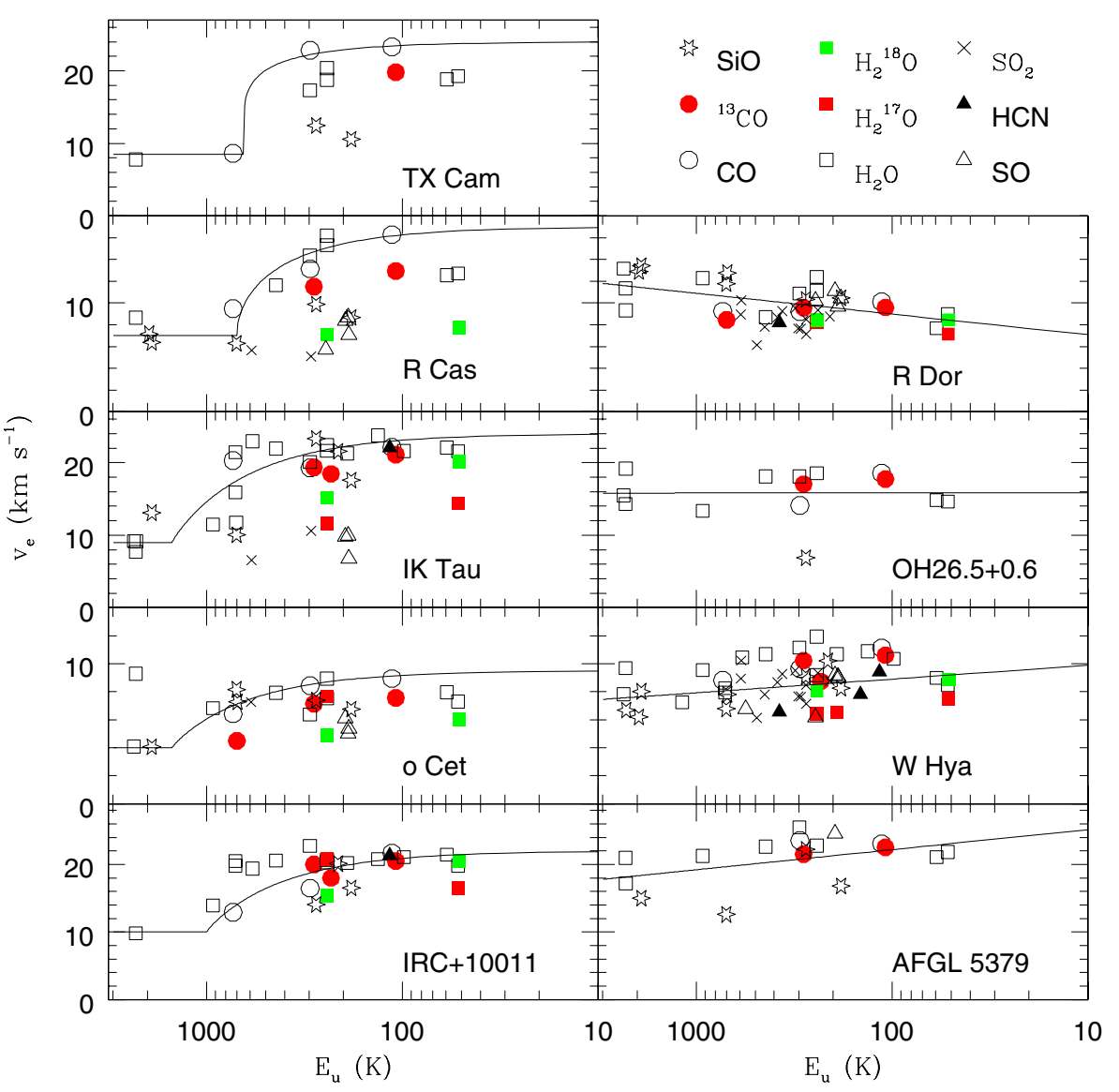

Fig. 6. Observed expansion velocity for molecular lines in our sample stars. more robust $\mathrm{CO}$ molecules. In some stars with a relatively low mass-loss rate, the measured velocities of $\mathrm{SiO} J=16-15$ and ${ }^{29} \mathrm{SiO} J=13-12$ lines also are smaller than those from $\mathrm{CO}$.

A number of stars show a clear trend of the high excitation lines having a smaller line width than low excitation ones (Fig. 6), consistent with a region where the molecules are then accelerated towards the terminal velocity observed in the ground-based low- $J$ CO observations. In order to qualitatively show that the wind is accelerated, we overplot a function similar to the dust-driven wind of Lamers \& Cassinelli (1999) but with a dependence on the energy rather than the radius:

$v=v_{0}+\left(v_{\mathrm{e}}-v_{0}\right)\left[1 .-E_{0} / E\right]^{\beta}$

where $v_{0}, v_{\mathrm{e}}$ and $E_{0}$ are arbitrary initial and expansion velocities in $\mathrm{km} \mathrm{s}^{-1}$ and energy in $\mathrm{K}$, respectively, and $\beta$ is a velocity exponent (Table 2). The parameters are chosen to follow the ${ }^{12} \mathrm{CO}$ transitions as a the main tracer for the expansion velocity. In a few cases, however, no clear jump in velocity between high and low excitation lines is seen, and a straight line can better describe the velocity field (right panels in Fig. 6). The fitted lines are least-square-fits to all the data points. In general, despite the smaller observed expansion velocity of $\mathrm{SO}, \mathrm{SO}_{2}, \mathrm{SiO}$ and less abundant isotopologues of $\mathrm{H}_{2} \mathrm{O}$ and $\mathrm{CO}$, the measured velocities of these species follow the overall trend of increasing velocity with decreasing excitation energy, i.e., increasing radius from the central star.

In the case of $\mathrm{R}$ Dor, however, the highly excited lines of both $\mathrm{H}_{2} \mathrm{O}$ and $\mathrm{SiO}$ indicate a larger expansion velocity in the inner part of the envelope than for the lower $J$-transitions of CO. This is counter-intuitive compared to what is expected of an accelerating dust-driven wind and may pose a challenge to detailed radiative transfer modelling of the stellar wind of $\mathrm{R}$ Dor. Also in
Table 2. Parameters describing the velocity profile for stars which show wind acceleration (Fig. 6).

\begin{tabular}{lcccc}
\hline \hline & $\begin{array}{c}v_{0} \\
\left(\mathrm{~km} \mathrm{~s}^{-1}\right)\end{array}$ & $\begin{array}{c}v_{\mathrm{e}} \\
\left(\mathrm{km} \mathrm{s}^{-1}\right)\end{array}$ & $\begin{array}{c}E_{0} \\
\mathrm{~K}\end{array}$ & $\beta$ \\
\hline TX Cam & 8.5 & 24.0 & 650. & 0.2 \\
R Cas & 7.0 & 17.0 & 700. & 0.5 \\
IK Tau & 9.0 & 24.0 & 1500. & 0.9 \\
$o$ Cet & 4.0 & 9.5 & 1500. & 0.9 \\
IRC+10011 & 10.0 & 22.0 & 1000. & 0.9 \\
\hline
\end{tabular}

AFGL 5379 and $\mathrm{OH} 26.5+0.6$, the expansion velocity of various lines suggests that the wind reaches the terminal velocity already in the innermost part, i.e., the acceleration zone is very small since the line widths are similar regardless of the excitation. For W Hya, the line widths increase linearly with the decreasing excitation energy. These stars demonstrate that the gasdust interaction does not seem to follow a simple momentum transfer previously used to describe the wind. To fully understand the observed line widths, a full radiative transfer must be performed including a velocity field and temperature profile.

\section{Summary}

We present the full dataset on O-rich AGB stars as part of the guaranteed time key program HIFISTARS to study the late stages of stellar evolution both kinematically and dynamically. We detect emission of 9 molecular species and their most common isotopologues. We find a trend of increasing line luminosity of $\mathrm{H}_{2} \mathrm{O}, \mathrm{CO}$ and $\mathrm{NH}_{3}$ with the mass-loss rate (Fig. 2) which tapers off for stars with a high mass-loss rate. Interestingly, the 
line ratios of high- $J$ CO relative to $\mathrm{CO} J=6-5$ are independent of the mass-loss rate (Fig. 3). This implies that the excitation of high- $J \mathrm{CO}$ is independent of the density, i.e., the lines are thermalized. Other species such as $\mathrm{H}_{2} \mathrm{O}$ and $\mathrm{NH}_{3}$ show a positive correlation with the mass-loss rate.

From the line brightness and shape, we conclude that the $\mathrm{H}_{2} \mathrm{O} 620.7 \mathrm{GHz}$ is masing in all three objects observed. The $970.3 \mathrm{GHz}$ and the $658.0 \mathrm{GHz}$ lines are masing in objects with relatively low $\left(\leq 10^{-5} M_{\odot} \mathrm{yr}^{-1}\right)$ mass-loss rates. These maser lines are generally significantly narrower than the thermal $\mathrm{H}_{2} \mathrm{O}$ lines.

Since the lines are well resolved, we can use the observed line width to derive the expansion velocity for each transition. In general, highly excited lines which originate close to the star have smaller expansion velocities compared to the lower excitation lines (Fig. 6). This is in agreement with the gas being dragged by dust grains as they are accelerated outward due to stellar radiation. However, from our observations, the acceleration zone differs in our sample, with stars with a high mass-loss rate showing high-excitation lines having velocity close to the terminal velocity while stars with a low mass-loss rate having a distinct low velocity region $\left(E_{u} \leq 10^{3} \mathrm{~K}\right)$. In R Dor, the highly excited lines show smaller observed line widths than the low excited lines. For this object, the gas appears to be decelerating as it moves away from the central star. A caveat is the optical depth which can affect the line profile. In order to calculate this effect, a full radiative transfer has to be performed in individual objects. This work has started and will be presented in the forth coming papers.

Acknowledgements. HCSS/HSpot/HIPE is a joint development (are joint developments) by the Herschel Science Ground Segment Consortium, consisting of ESA, the NASA Herschel Science Center, and the HIFI, PACS and SPIRE consortia. K.J., F.S., M.M., and H.O. acknowledge funding from the Swedish National Space Board. This work has been partially supported by the Spanish MICINN, within the program CONSOLIDER INGENIO 2010, under grant "Molecular Astrophysics: The Herschel and Alma Era ASTROMOL" (ref.: CSD2009-00038). R.Sz. and M.Sch. acknowledge support from grant N 203581040 from Polish MNiSW. J.C. thanks funding from MICINN, grant AYA2009-07304. This research was performed, in part, through a JPL contract funded by the National Aeronautics and Space Administration. We would like to thank the referee for his/her careful reading and suggestions which improved the manuscript.

\section{References}

Barlow, M. J., Nguyen-Q-Rieu, Truong-Bach, et al. 1996, A\&A, 315, L241

Bowen, G. H. 1988, ApJ, 329, 299

Bujarrabal, V., Fuente, A., \& Omont, A. 1994, A\&A, 285, 247

Bujarrabal, V., Alcolea, J., Soria-Ruiz, R., et al. 2010, A\&A, 521, L3

Chapman, J. M., Sivagnanam, P., Cohen, R. J., \& Le Squeren, A. M. 1994, MNRAS, 268, 475

Cherchneff, I. 2006, A\&A, 456, 1001

de Beck, E., Decin, L., de Koter, A., et al. 2010, A\&A, 523, A18

de Graauw, T., Helmich, F. P., Phillips, T. G., et al. 2010, A\&A, 518, L6

Decin, L., Hony, S., de Koter, A., et al. 2006, A\&A, 456, 549

Decin, L., Agúndez, M., Barlow, M. J., et al. 2010a, Nature, 467, 64

Decin, L., Justtanont, K., de Beck, E., et al. 2010b, A\&A, 521, L4

Goldreich, P., \& Scoville, N. 1976, ApJ, 205, 144

Habing, H. J. 1996, A\&ARv, 7, 97

Habing, H. J., Tignon, J., \& Tielens, A. G. G. M. 1994, A\&A, 286, 523

Harwit, M., Houde, M., Sonnentrucker, P., et al. 2010, A\&A, 521, L51

Hasegawa, T. I., Kwok, S., Koning, N., et al. 2006, ApJ, 637, 791

Hoefner, S., \& Dorfi, E. A. 1992, A\&A, 265, 207

Hoefner, S., \& Dorfi, E. A. 1997, A\&A, 319, 648

Höfner, S. 2008, A\&A, 491, L1

Justtanont, K., Skinner, C. J., \& Tielens, A. G. G. M. 1994, ApJ, 435, 852

Justtanont, K., Olofsson, G., Dijkstra, C., \& Meyer, A. W. 2006, A\&A, 450, 1051

Justtanont, K., Decin, L., Schöier, F. L., et al. 2010, A\&A, 521, L6

Lamers, H. J. G. L. M., \& Cassinelli, J. P. 1999, Introduction to Stellar Winds (Cambridge, UK: Cambridge University Press)

Maercker, M., Schöier, F. L., Olofsson, H., et al. 2009, A\&A, 494, 243

Mattsson, L., Wahlin, R., Höfner, S., \& Eriksson, K. 2008, A\&A, 484, L5

Melnick, G. J., Neufeld, D. A., Ford, K. E. S., Hollenbach, D. J., \& Ashby, M. L. N. 2001, Nature, 412, 160

Menten, K. M., Wyrowski, F., Alcolea, J., et al. 2010, A\&A, 521, L7

Neufeld, D. A., \& Melnick, G. J. 1991, ApJ, 368, 215

Neufeld, D. A., Chen, W., Melnick, G. J., et al. 1996, A\&A, 315, L237

Neufeld, D. A., González-Alfonso, E., Melnick, G., et al. 2011, ApJ, 727, L29

O'Dwyer, I. J., Chu, Y., Gruendl, R. A., Guerrero, M. A., \& Webbink, R. F. 2003, AJ, 125, 2239

Olofsson, H., Lindqvist, M., Nyman, L.-A., \& Winnberg, A. 1998, A\&A, 329, 1059

Omont, A., Lucas, R., Morris, M., \& Guilloteau, S. 1993, A\&A, 267, 490

Pilbratt, G. L., Riedinger, J. R., Passvogel, T., et al. 2010, A\&A, 518, L1

Ramstedt, S., Schöier, F. L., Olofsson, H., \& Lundgren, A. A. 2008, A\&A, 487, 645

Schöier, F. L., Maercker, M., Justtanont, K., et al. 2011, A\&A, 530, A83

Sevenster, M. N., van Langevelde, H. J., Moody, R. A., et al. 2001, A\&A, 366, 481

Woitke, P. 2006, A\&A, 460, L9

Yamamura, I., de Jong, T., Onaka, T., Cami, J., \& Waters, L. B. F. M. 1999, A\&A, 341, L9

Pages 8 to 21 are available in the electronic edition of the journal at http: //www . aanda. org 


\section{Appendix A: Spectra}

In this section, we present the observed spectra of O-rich AGB stars observed in the HIFISTARS guaranteed time program, along with the measured line intensities.
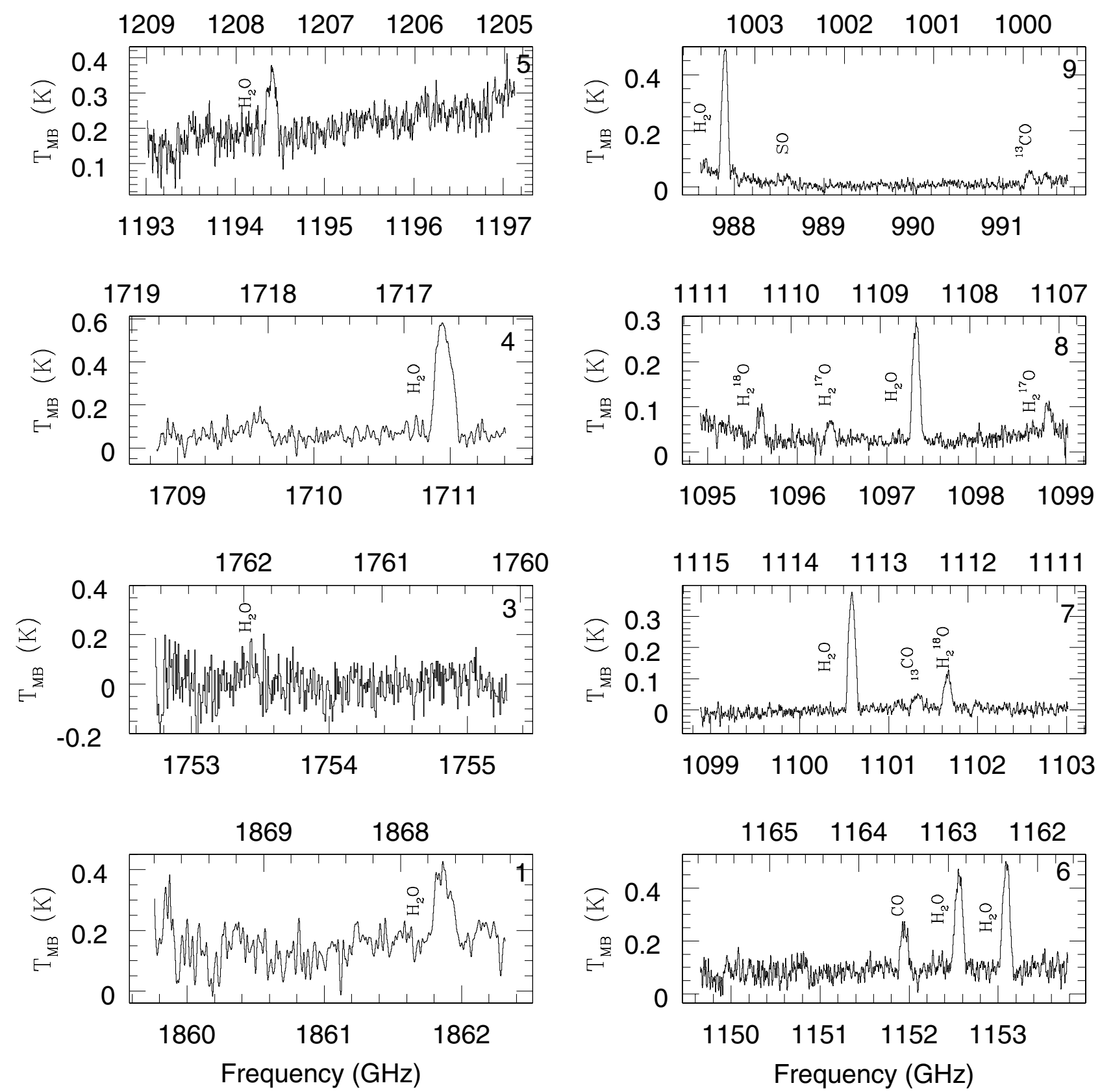

Fig. A.1. HIFI spectra of IRC+10011. For details of the lines detected, see Table A.1. 

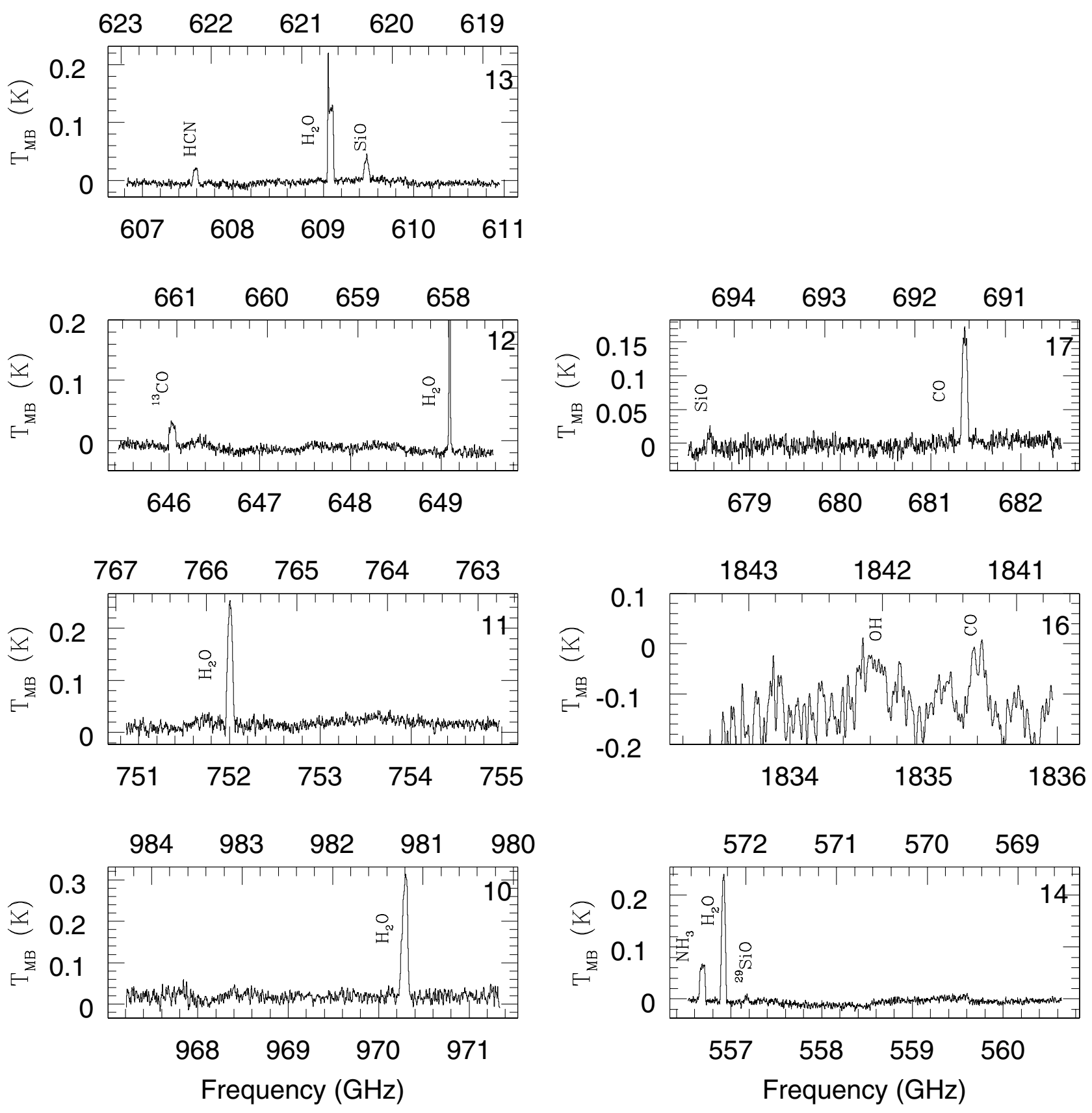

Fig. A.2. HIFI spectra of IRC +10011 , continued. 

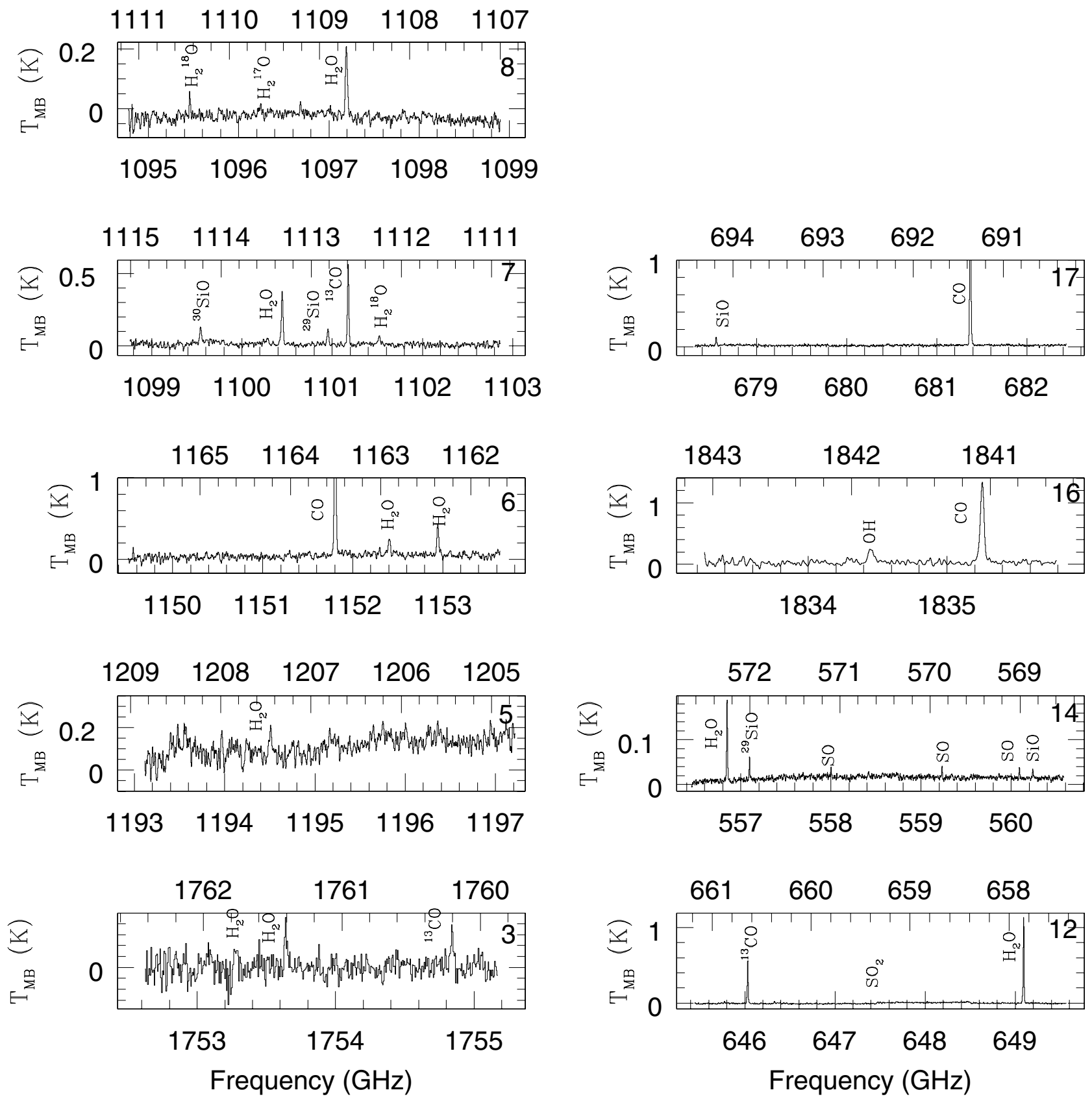

Fig. A.3. HIFI spectra of $o$ Cet. 
K. Justtanont et al.: Herschel/HIFI observations of O-rich AGB stars: molecular inventory
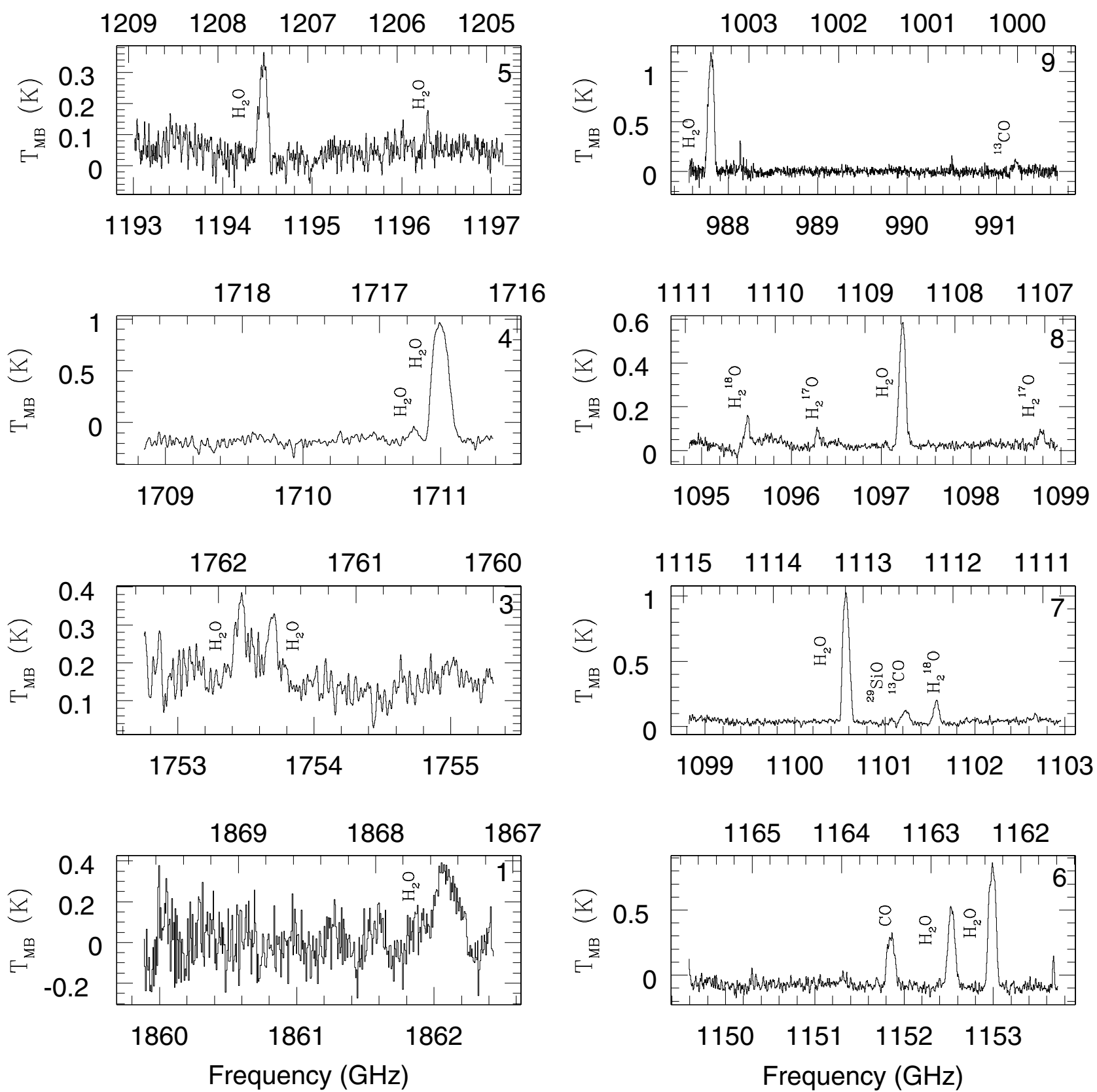

Fig. A.4. HIFI spectra of IK Tau. 

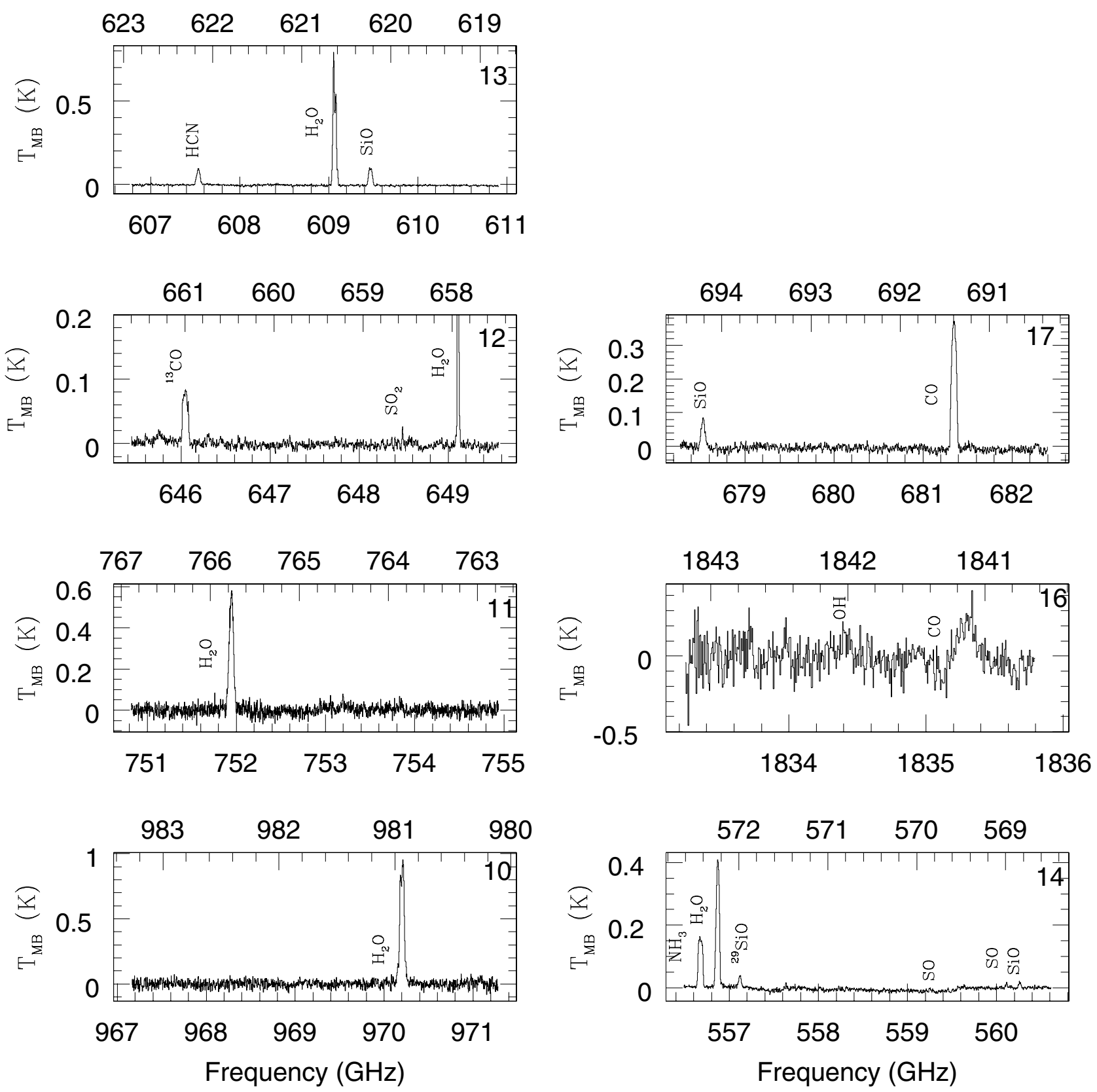

Fig. A.5. HIFI spectra of IK Tau, continued. 
K. Justtanont et al.: Herschel/HIFI observations of O-rich AGB stars: molecular inventory
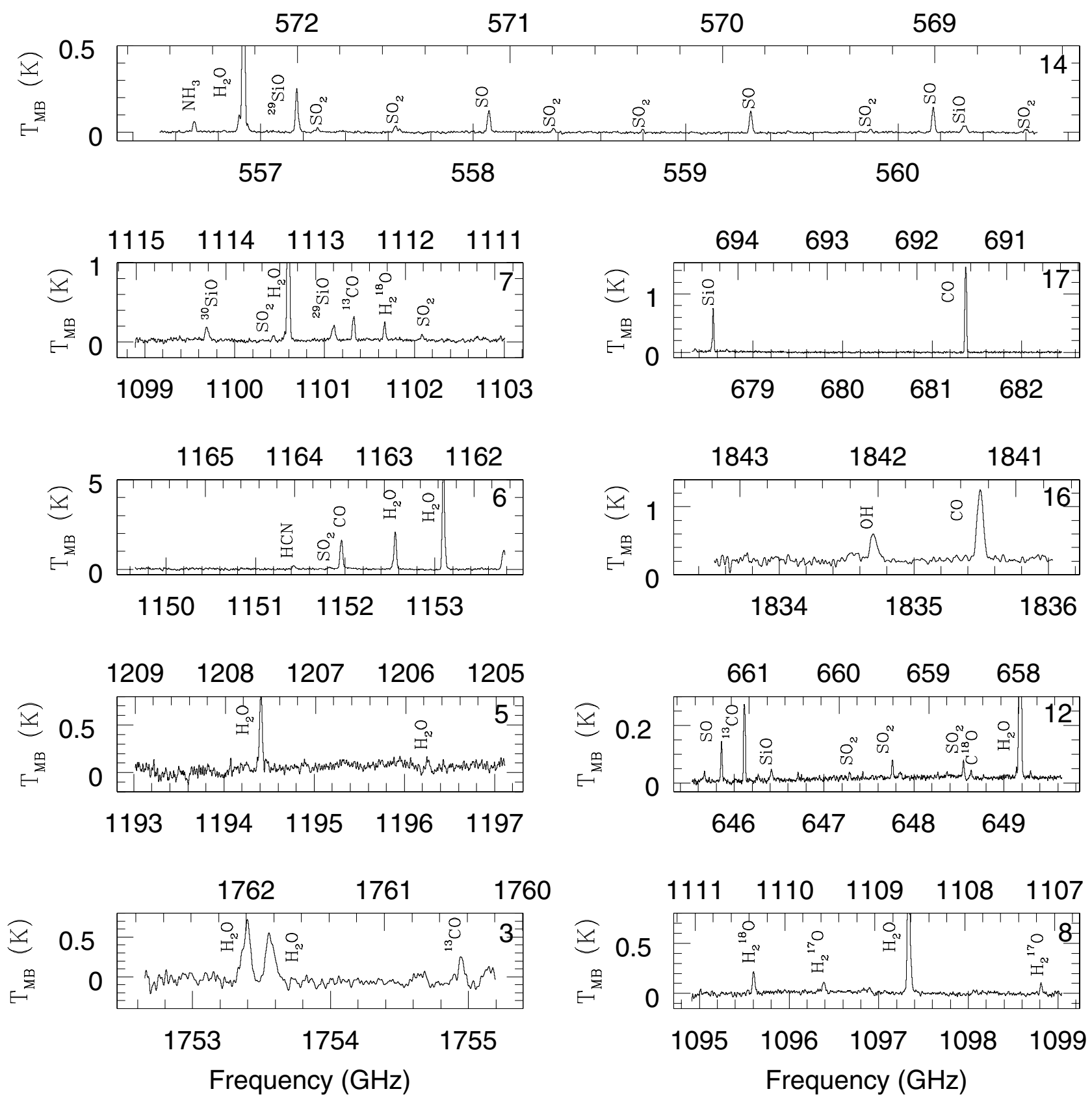

Fig. A.6. HIFI spectra of R Dor. 

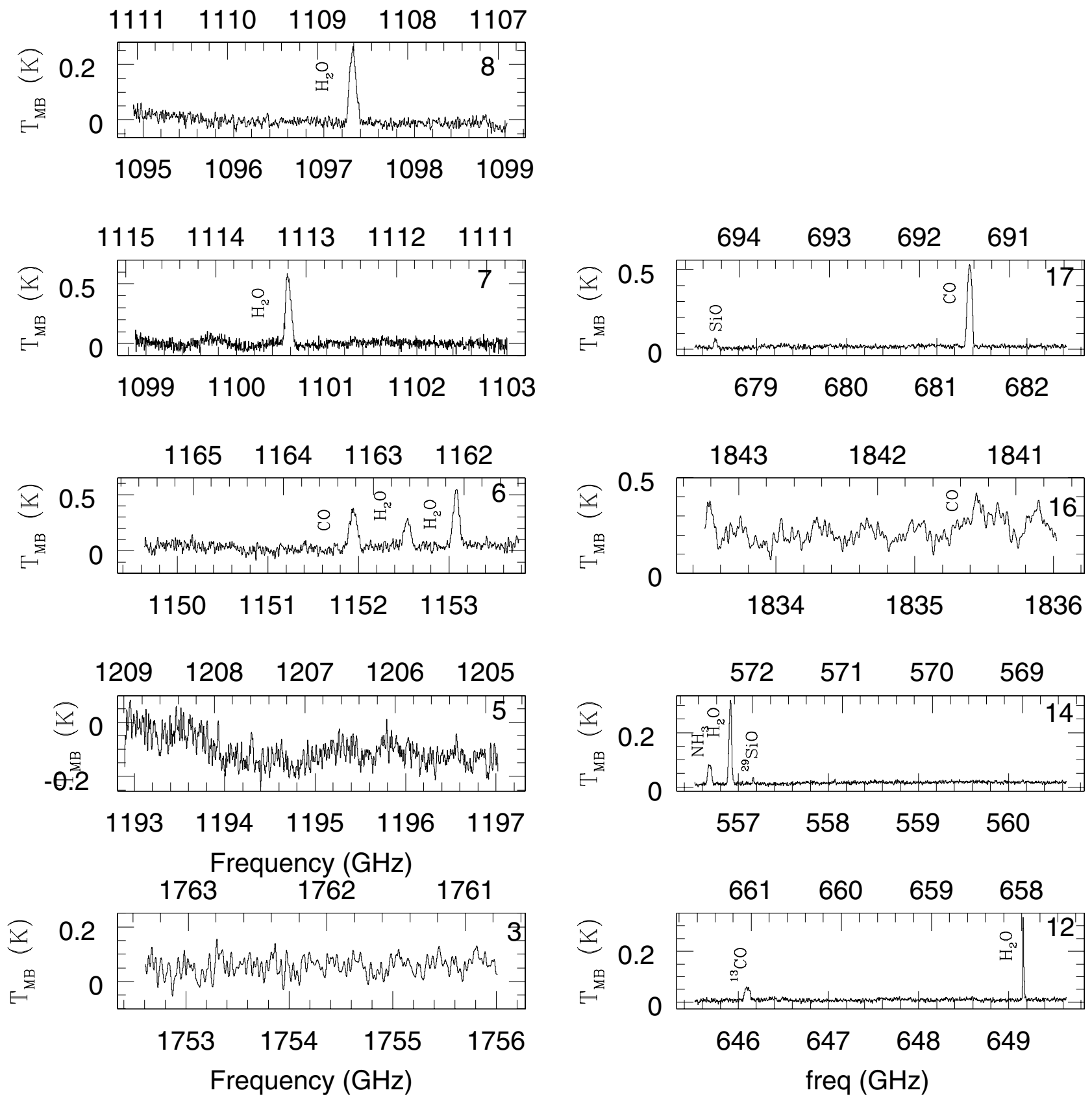

Fig. A.7. HIFI spectra of TX Cam. 
K. Justtanont et al.: Herschel/HIFI observations of O-rich AGB stars: molecular inventory
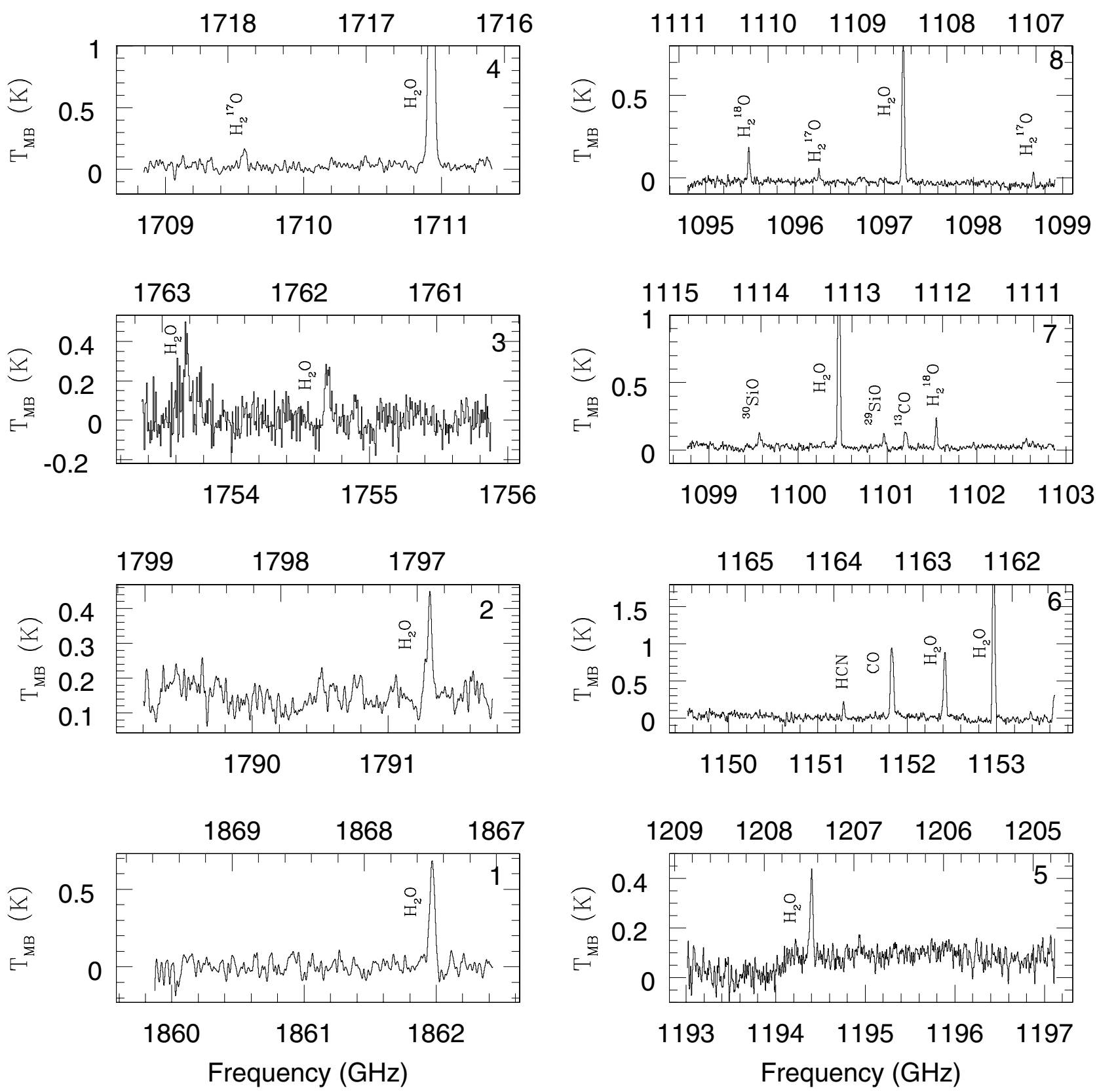

Fig. A.8. HIFI spectra of WHya. 

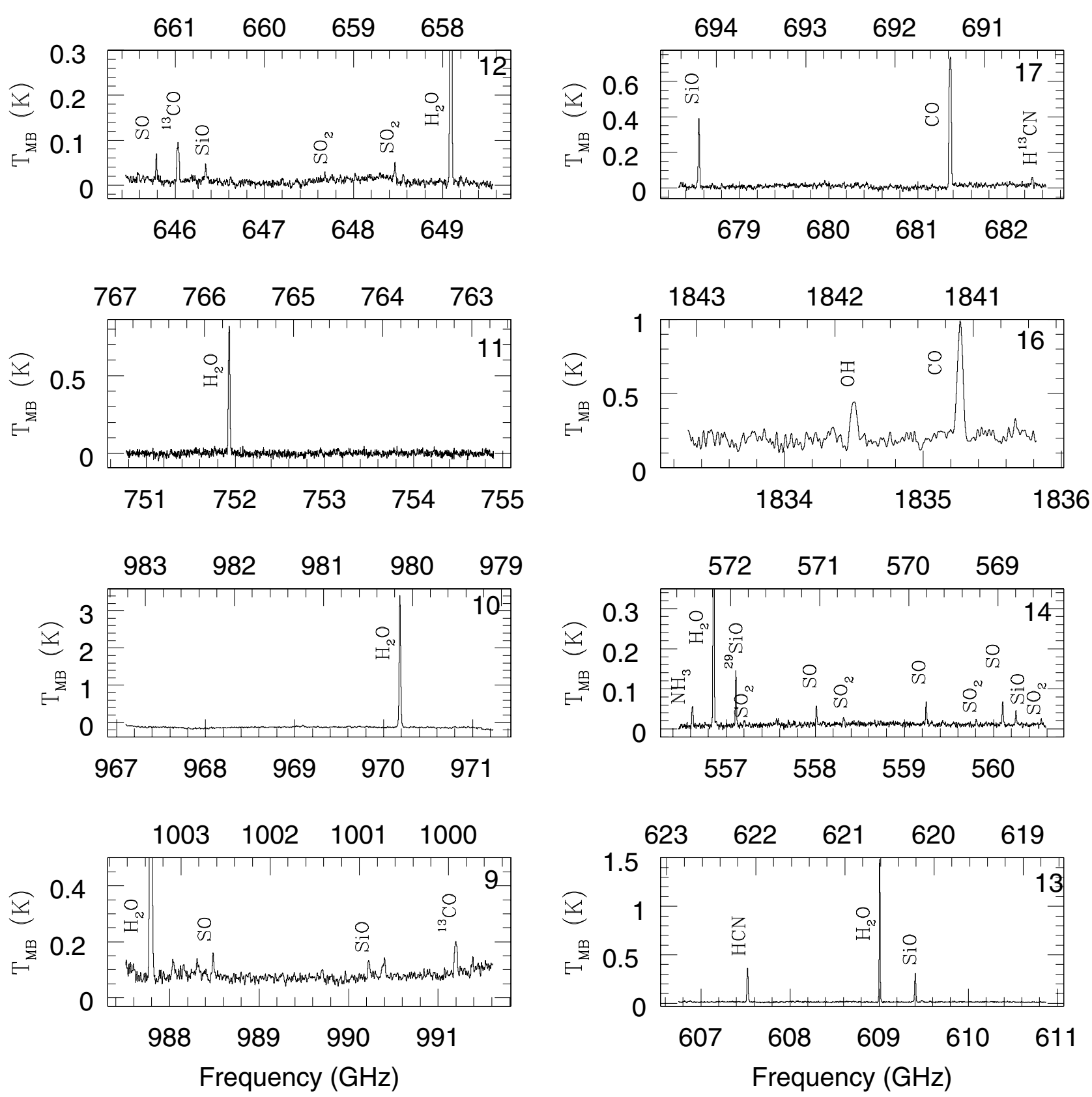

Fig. A.9. HIFI spectra of WHya, continued. 
K. Justtanont et al.: Herschel/HIFI observations of O-rich AGB stars: molecular inventory
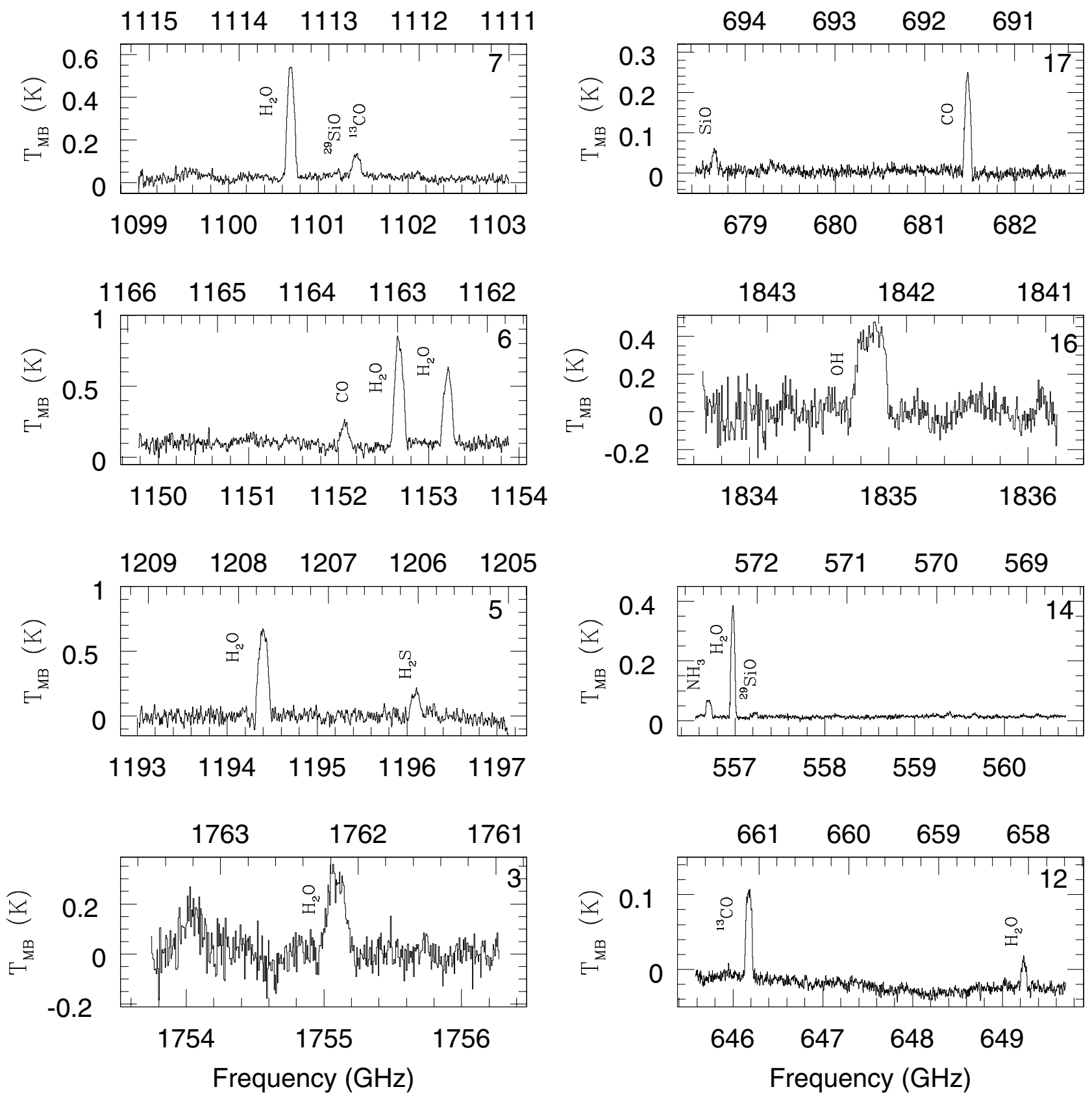

Fig. A.10. HIFI spectra of AFGL 5379. 

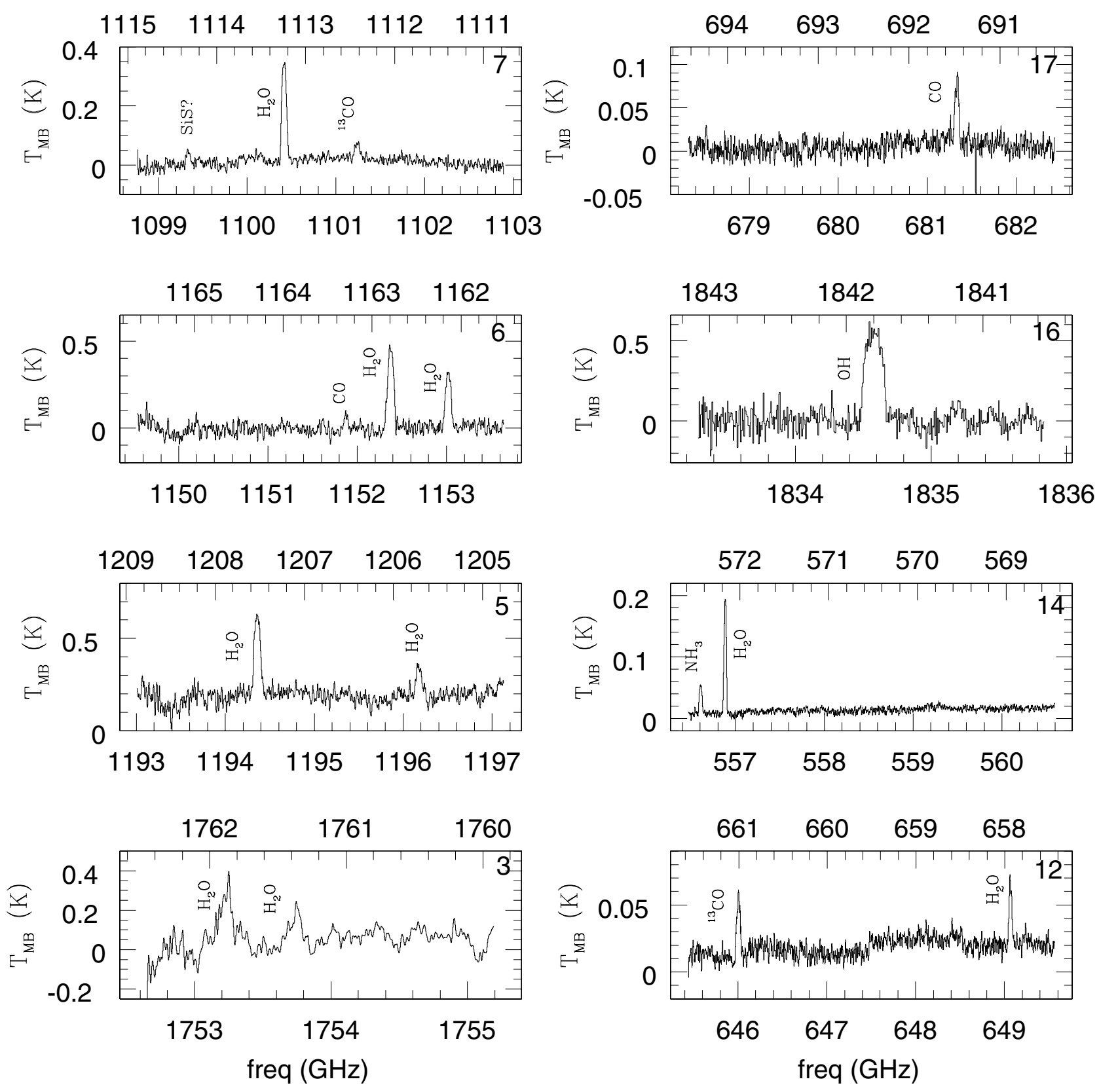

Fig. A.11. HIFI spectra of $\mathrm{OH} 26.5+0.6$. 

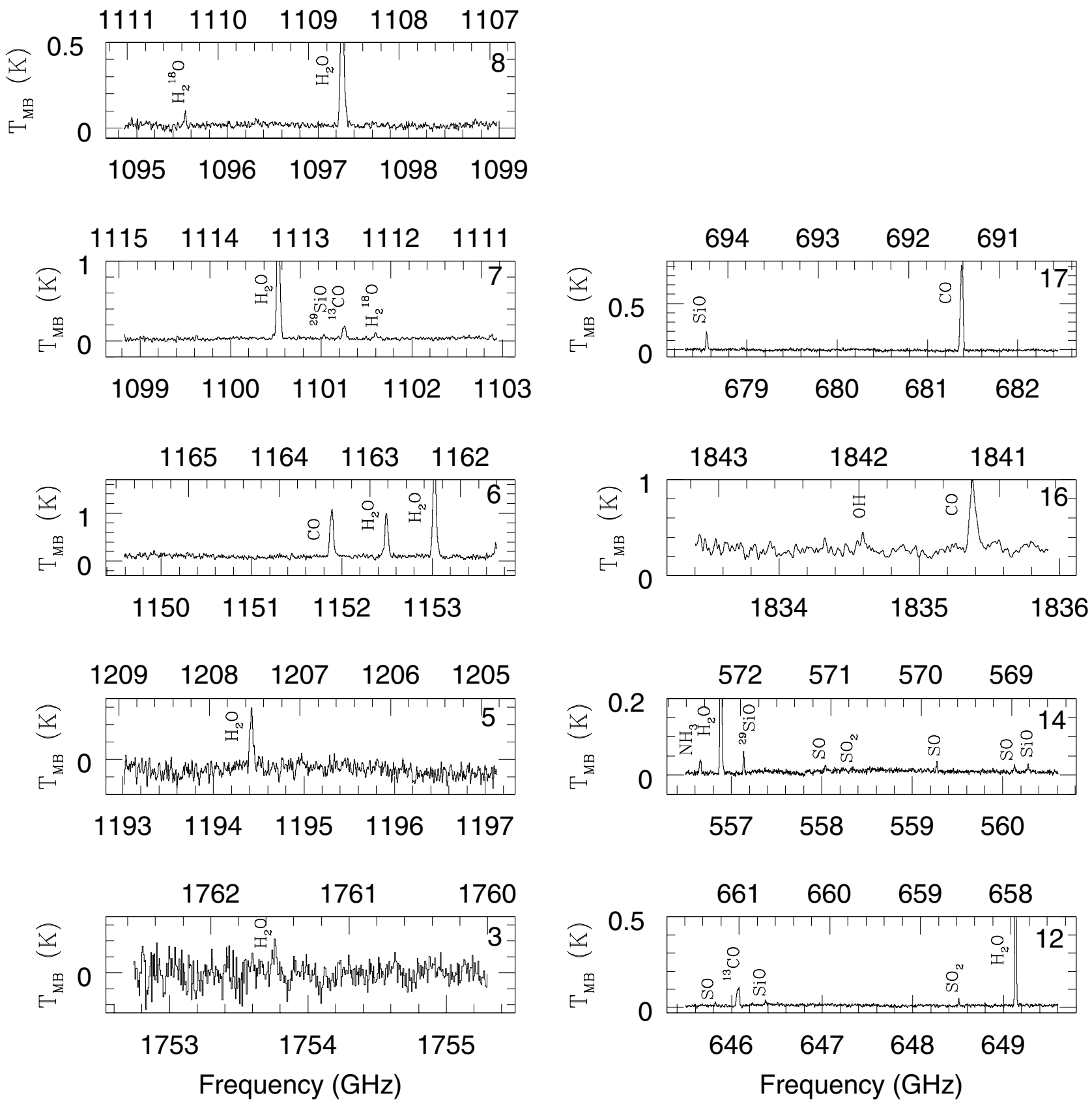

Fig. A.12. HIFI spectra of R Cas. 


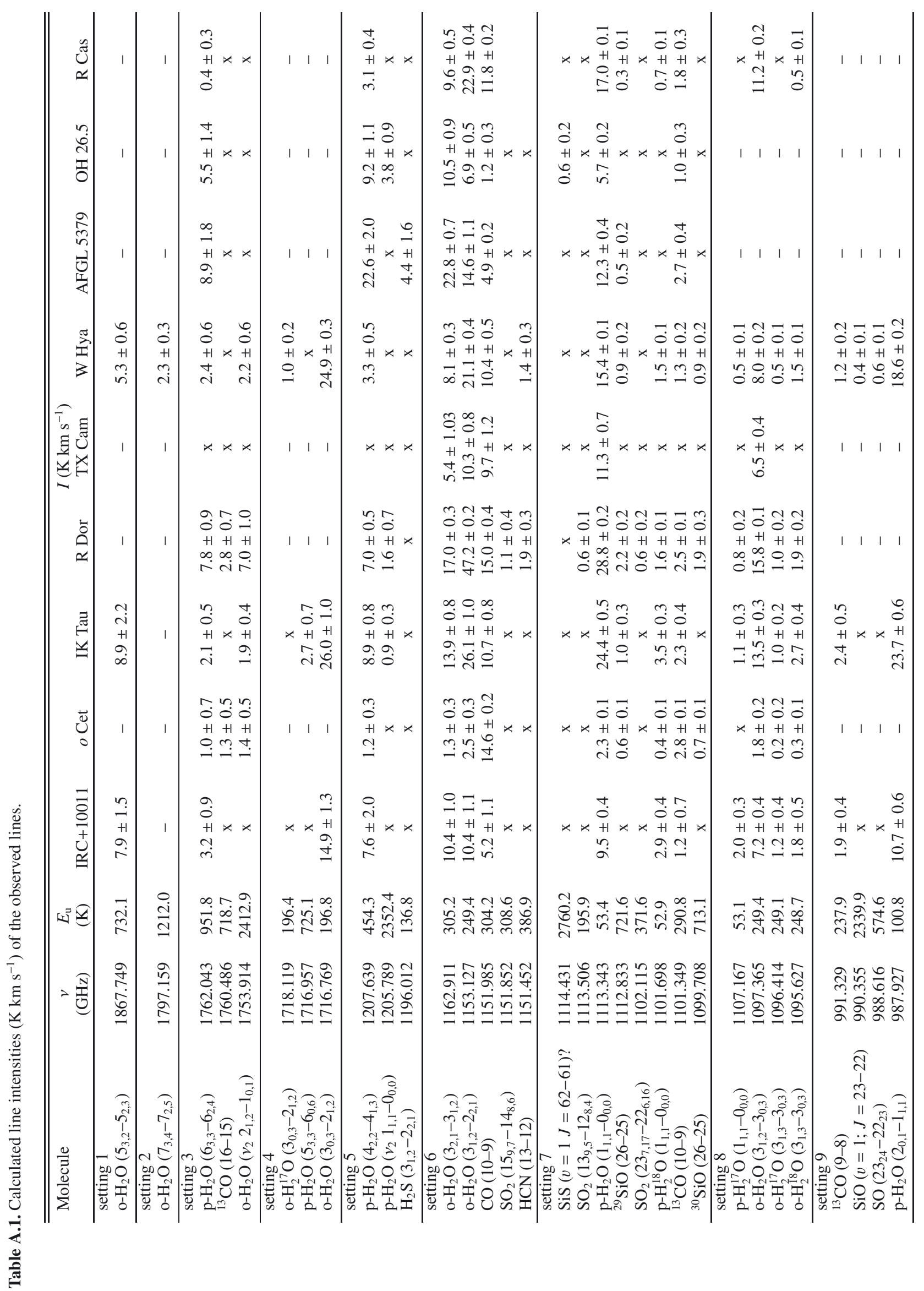


K. Justtanont et al.: Herschel/HIFI observations of O-rich AGB stars: molecular inventory

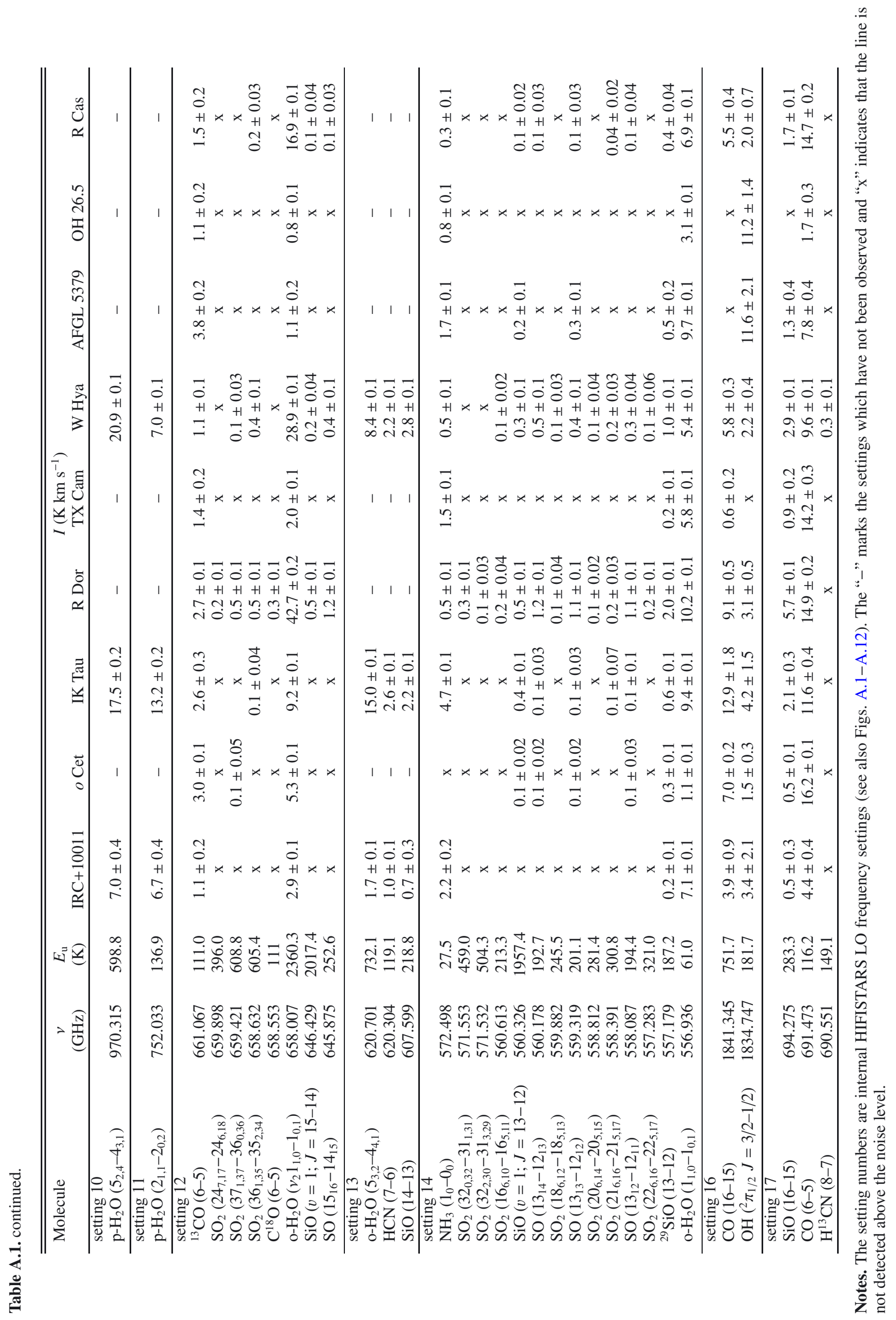

